\title{
Mitigating oxygen stress enhances aged mouse hematopoietic stem cell numbers and function
}

\author{
Maegan L. Capitano, ${ }^{1}$ Safa F. Mohamad, ${ }^{1}$ Scott Cooper, ${ }^{1}$ Bin Guo, ${ }^{1}$ Xinxin Huang, ${ }^{1,2}$ Andrea M. Gunawan, ${ }^{3}$ Carol Sampson, ${ }^{3}$ \\ James Ropa, ${ }^{1}$ Edward F. Srour, ${ }^{1,3,4}$ Christie M. Orschell, ${ }^{3}$ and Hal E. Broxmeyer ${ }^{1}$ \\ 'Department of Microbiology and Immunology, Indiana University School of Medicine, Indianapolis, Indiana, USA. ${ }^{2}$ Zhongshan-Xuhui Hospital and Shanghai Key Laboratory of Medical Epigenetics, \\ Institutes of Biomedical Sciences, Fudan University, Shanghai, China. ${ }^{3}$ Department of Medicine, Indiana University School of Medicine, Indianapolis, Indiana, USA. ${ }^{4}$ Department of Pediatrics, \\ Indiana University School of Medicine, Indianapolis, Indiana, USA.
}

\begin{abstract}
Bone marrow (BM) hematopoietic stem cells (HSCs) become dysfunctional during aging (i.e., they are increased in number but have an overall reduction in long-term repopulation potential and increased myeloid differentiation) compared with young HSCs, suggesting limited use of old donor BM cells for hematopoietic cell transplantation (HCT). BM cells reside in an in vivo hypoxic environment yet are evaluated after collection and processing in ambient air. We detected an increase in the number of both young and aged mouse BM HSCs collected and processed in $3 \% \mathrm{O}_{2}$ compared with the number of young BM HSCs collected and processed in ambient air $\left(21 \% \mathrm{O}_{2}\right)$. Aged BM collected and processed under hypoxic conditions demonstrated enhanced engraftment capability during competitive transplantation analysis and contained more functional HSCs as determined by limiting dilution analysis. Importantly, the myeloid-to-lymphoid differentiation ratio of aged BM collected in $3 \% \mathrm{O}_{2}$ was similar to that detected in young BM collected in ambient air or hypoxic conditions, consistent with the increased number of common lymphoid progenitors following collection under hypoxia. Enhanced functional activity and differentiation of old BM collected and processed in hypoxia correlated with reduced "stress" associated with ambient air BM collection and suggests that aged BM may be better and more efficiently used for HCT if collected and processed under hypoxia so that it is never exposed to ambient air $\mathrm{O}_{2}$.
\end{abstract}

\section{Introduction}

Aging is an inevitable process associated with the deterioration of normal physiological functions. This includes hematopoietic changes such as increased numbers of hematopoietic stem cells (HSCs) that have decreased engraftment capability in lethally irradiated mice (1-5) and biased differentiation (i.e., more myeloid but fewer lymphoid cells produced) $(1,3,5-9)$. These changes to hematopoiesis associated with aging HSCs limit the use of aged bone marrow (BM) as a donor source for both allogeneic and autologous hematopoietic cell transplantation (HCT).

The BM niche nurtures the survival and proliferation of HSCs, hematopoietic progenitor cells (HPCs), and hematopoiesis during adult life. The area of oxygen sensing has recently been recognized with the Nobel Prize $(10,11)$. This niche is hypoxic $(\sim 1 \%-5 \%$ $\mathrm{O}_{2}$ tension) compared with ambient air $\left(21 \% \mathrm{O}_{2}\right)$. Proliferation of HSCs and HPCs ex vivo is more efficient when cells are cultured in a hypoxic environment of approximately $5 \%$ oxygen tension than when cultured in ambient air $\mathrm{O}_{2}$ (12-20). However, all studies comparing young and aged hematopoiesis have been based on the numbers and activity of HSCs and HPCs first collected and processed in ambient air, regardless of whether the BM cells were

Conflict of interest: The authors have declared that no conflict of interest exists. Copyright: (5) 2021, American Society for Clinical Investigation.

Submitted: May 11, 2020; Accepted: September 24, 2020; Published: January 4, 2021.

Reference information: / Clin Invest. 2021;131(1):e140177.

https://doi.org/10.1172/JCl140177. subsequently cultured and studied in ambient air or under hypoxic $\mathrm{O}_{2}$ tension. Hematopoietic cell populations from BM of young adult mice and human cord blood demonstrated a large loss of HSC numbers with an associated increase in HPC numbers within minutes of exposure of these cells to ambient air, via a phenomenon we termed extra physiologic oxygen shock/stress (EPHOSS) (21-23). Loss of HSCs was due to EPHOSS-induced rapid differentiation of HSCs into HPCs, events mediated through a mitochondrial permeability transition pore/p53/cyclophilin D (MPTP/ p53/cyclophilin D) axis associated with HIF-1 $\alpha$ and the hypoxamir miR210. This involved a rapid increase in the release of ROS due to ambient air exposure-induced opening of the MPTP (21-23). Thus, collection and processing of these cells in $3 \% \mathrm{O}_{2}$, such that they were never exposed to ambient air, resulted in increased numbers of phenotypic and functional HSCs (23).

The ability of hypoxia collection/processing of young mouse $\mathrm{BM}$ to increase HSC numbers and function led us to reevaluate primitive $\mathrm{BM}$ cells from aged mice in the context of collection and processing at $3 \% \mathrm{O}_{2}$ versus ambient air in an effort to determine whether aged BM might potentially be used for HCT. We hypothesized that the collection and processing of aged BM (from mice 20-28 months of age) under hypoxic conditions would manifest in improved HSC function closer to that of HSCs collected from young ( 8- to 12-week-old) mice and thus engraft more efficiently following transplantation. We demonstrate here that collection and processing of older mouse $\mathrm{BM}$ under $3 \% \mathrm{O}_{2}$ resulted in increased aged mouse HSC function, normalization 
of the myeloid/lymphoid cell ratio, and increased numbers of common lymphoid progenitors (CLPs), effects associated with decreased total and mitochondrial ROS and decreased expression of stress-induced proteins in aged HSCs. We hypothesize that collecting and processing BM from aged individuals under hypoxic conditions may preserve or enhance HSC function enough so that the HSCs could be used for clinical HCT, and potentially for the still-emerging field of regenerative medicine.

\section{Results}

Hypoxic collection/processing of aged mouse donor BM alters the phenotypic makeup of HSC and HPC compartments. We deciphered the effects of hypoxic versus air collection and processing on the phenotypic makeup of donor BM cells from young versus old mice prior to the use of these cells in transplantation experiments. We performed all collections and assays as reported previously (23), taking great care to ensure that everything used for hypoxic collections (media, plasticware, glassware, syringes, antibodies, etc.) was equilibrated at $3 \% \mathrm{O}_{2}$ for at least 18 hours prior to and during removal and processing of the cells. Cells collected in hypoxia were split into 2 parts (Figure 1A): one was left and processed in hypoxia (so that the cells were never exposed to ambient air), and the other was acclimated for 1 hour in ambient air (after collection in $3 \% \mathrm{O}_{2}$ ) and then processed in ambient air. We had previously shown that there was no difference in the number or function of BM HSCs or HPCs of young mice collected directly in ambient air versus being removed from hypoxia to ambient air for 1 hour and subsequently processed in ambient air (23).

Although we detected no differences in the number of nucleated cells/femur between young (8- to 12-week-old) and old (20to 28-month-old) C57BL/6 mice (Supplemental Figure 1A; supplemental material available online with this article; https://doi. org/10.1172/JCI140177DS1), we found significant differences in these age groups in terms of the number of ambient air-acclimated HSCs (long-term [LT] HSCs and short-term [ST] HSCs), and HPCs (multipotent progenitors [MPPs], common myeloid progenitors [CMPs], granulocyte-macrophage progenitors [GMPs], megakaryocyte erythrocyte progenitors [MEPs], and CLPs) as defined by phenotype (Figure 1 and Supplemental Figure 1, B and C). Ambient air-acclimated HSCs and all but the CLP populations of HPCs were significantly increased in old mice (Figure 1, B-E, and Supplemental Figure 1, B and C), while CLPs were significantly decreased in old mice (Figure $1 \mathrm{~F}$ ). We noted similar results for BM HSCs and HPCs with another mouse strain (CB6) (Supplemental Figure 2). These results for ambient air-acclimated/processed BM are similar to the values reported by others $(1,2,4-9$, 24). However, comparison of the cell populations from these same mice collected and processed under hypoxia $\left(3 \% \mathrm{O}_{2}\right)$ resulted in large differences from ambient air-acclimated cells (Figure 1, B-F, and Supplemental Figure 2). As we reported previously (23), young mouse BM LT-HSCs were significantly increased, whereas many HPC populations were decreased in hypoxia collection and processing compared with ambient air cohorts. We observed similar changes between hypoxic versus ambient air processing for old mice, but the differences were diminished (e.g., 3.6-fold increase for young vs. 1.8-fold increase for old LT-HSCs). One important and notable exception was that CLPs from old mice (in both mouse strains examined) showed significantly increased numbers of CLPs when BM cells were collected and processed under hypoxic conditions (Figure $1 \mathrm{~F}$ and Supplemental Figure $2 \mathrm{G}$ ).

Enhanced engraftment efficiency of old mouse BM HSCs collected and processed in hypoxia. The number of phenotypic HSCs and HPCs does not always recapitulate functional activities, especially under stress conditions (e.g., stresses of ambient airinduced EPHOSS) (25-27). We assessed the functional capacities of HSCs by in vivo chimerism and limiting dilution analysis of donor (C57BL/6; CD45.1-CD45.2+) cells from young and old mice in a competitive assay for both ambient air- and hypoxiacollected/processed cells. Lethally irradiated B6 Boy/J F1 $\left(\mathrm{CD} 45.1^{+} \mathrm{CD} 45.2^{+}\right)$recipient mice were injected i.v. with donor cells and a constant number of ambient air-collected competitor (Boy/J; CD 45.1 $\left.{ }^{+} \mathrm{CD} 45.2^{-}\right)$cells. Hypoxia-collected cells were injected i.v. into mice in a holder within the hypoxic chamber, where they could breathe ambient air, but their tail (where the cells were injected) was in hypoxia (23). Thus, hypoxia-collected cells were never exposed ex vivo to ambient air (Figure 2A). Ambient air-acclimated cells were injected into mice in ambient air. At 6 months, ambient air-acclimated young donor cells showed greater engraftment of peripheral blood (PB) (Figure 2B) and BM (Figure 2C) than did ambient air-collected cells from the old mice, consistent with other reports in the literature $(1,5,28,29)$. Although both young and old hypoxia-collected BM cells showed enhanced engraftment compared with ambient air-collected BM cells, we found that the differences were greater for BM cells from old mice (see the BM cells from primary recipients at 6 months, Figure 2C; 2.1-fold [young] vs. 4.1-fold [old] increase in engraftment when collected in hypoxia vs. ambient air). The engraftment of old BM collected under hypoxic conditions was equal to that of young BM acclimated to ambient air.

For secondary transplants, BM from our primary recipients was collected and processed in ambient air conditions (see the experimental procedures outlined in Figure 2A). When we first examined engraftment after secondary transplantation, we observed a significant increase in engraftment when the aged donor BM from the primary transplant was isolated under hypoxic conditions compared with aged BM isolated under ambient air conditions (2.1-fold increase; Figure 2D). However, this effect was not long lasting, and the enhanced engraftment of hypoxia-isolated/ processed primary recipient aged BM was reduced by month 2 (Figure 2E) and no longer significantly different from the engraftment of ambient air-isolated/processed primary recipient BM from aged mice by month 4 (Figure 2, F and G). These data suggest that the primary isolation and processing of donor BM under hypoxia and the subsequent engraftment into young recipient mice "rejuvenated" the aged HSCs and HPCs but that this slight rejuvenation was not permanent.

We performed limiting dilution analysis to calculate competitive repopulating units (CRU), a measure of functional HSC numbers, for the primary transplants. The CRU numbers were like those for BM engraftment chimerism studies (Figure $2 \mathrm{H}$ and Supplemental Table 1), with ambient air acclimation and processing showing a 4.3-fold decrease in CRU between old and young mouse $\mathrm{BM}$. Although both young and old BM manifested increased numbers of CRU in hypoxia- versus ambient air-collected/processed 
A

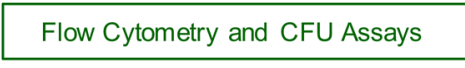

Isolate BM cells from young and old mice at $3 \% \mathrm{O}_{2}$

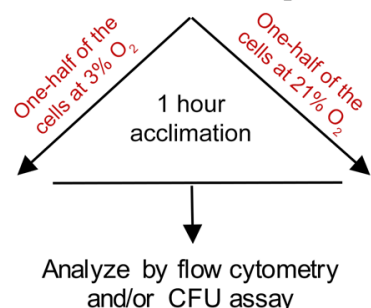
and/or CFU assay
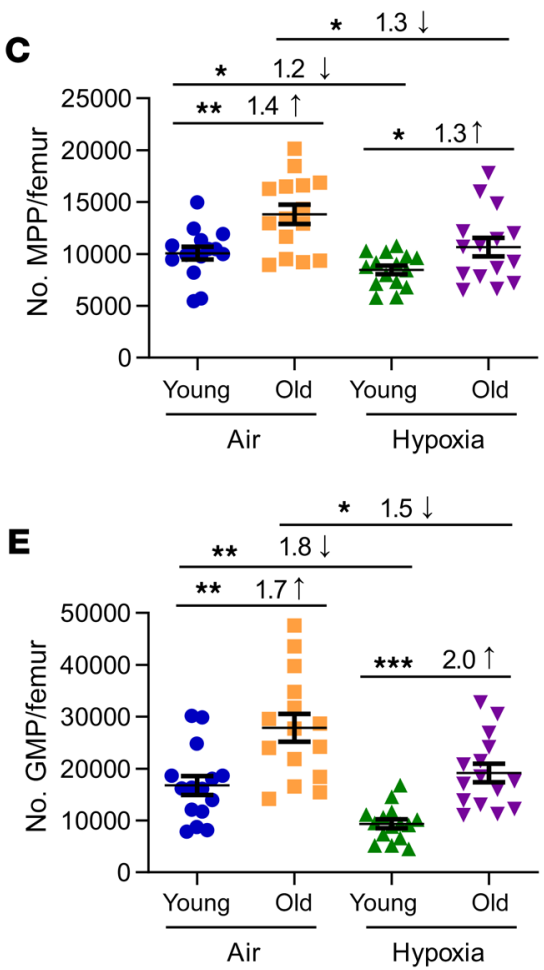

B

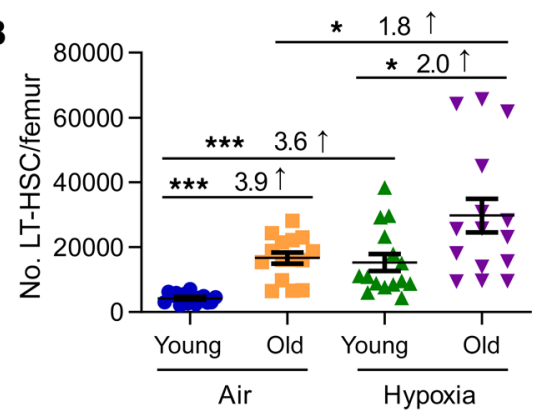

D

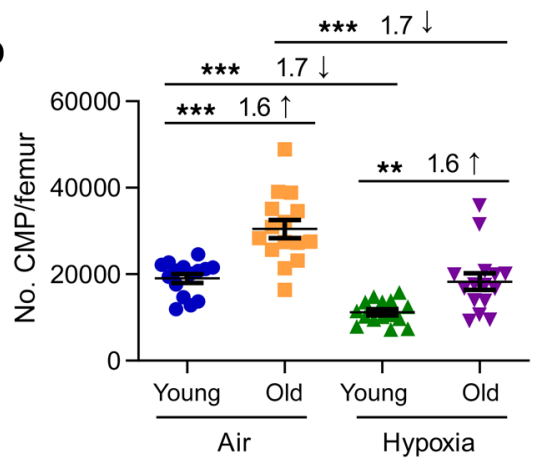

$\mathbf{F}$

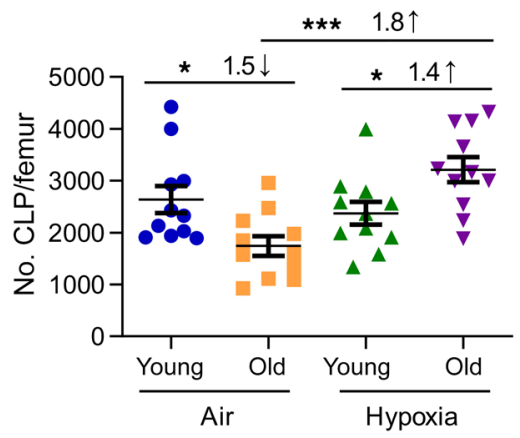

Figure 1. Phenotyping of young and old C57BL/6 mouse BM HSCs and HPCs collected under hypoxia and processed under ambient air ( $21 \%$ $\mathrm{O}_{2}$ ) versus hypoxia $\left(3 \% \mathrm{O}_{2}\right)$. (A) In a hypoxic glove box (acclimated to $3 \% \mathrm{O}_{2}$ for 18 hours), femurs from young (8- to -12-week-old) and old (20- to

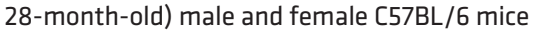
were flushed in sterile PBS, counted, and split in half so that one half remained under hypoxia and the other half were removed from hypoxia and acclimated to ambient air for 1 hour. Nucleated cells were analyzed. (B) Number of LT-HSCs (Lin-Sca-1+ ${ }^{+} \mathrm{C}-\mathrm{Kit}^{+} \mathrm{Flt} 3^{-}{ }^{-}$CD34-) per femur. (C) Number of MPPs ( $\left.\mathrm{Lin}^{-} \mathrm{Sca}-1^{+} \mathrm{C}-\mathrm{Kit}^{+} \mathrm{Flt3} 3^{+} \mathrm{CD} 34^{+}\right)$per femur.

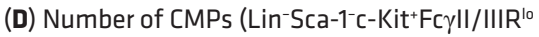
CD34 $4^{+}$) per femur. (E) Number of GMPs (Lin'Sca-1c-Kit ${ }^{+}$Fcyll/IIIR ${ }^{\text {hic }}$ (D34 $4^{+}$) per femur. (F) Number of CLPs (Lin-Sca- ${ }^{10} \mathrm{c}-\mathrm{Kit}^{\mathrm{l}} \mathrm{F} / \mathrm{t} 3^{+} \mathrm{IL}-7 \mathrm{R}^{+}$) per femur. (B-F) Data represent the mean \pm SEM for 11-15 C57BL/6 mice from 3-4 experiments. ${ }^{*} P<0.05$, ${ }^{* *} P<0.01$, and ${ }^{* *} P<0.001$, by 1-way ANOVA with post hoc Tukey's multiple-comparison test. cells, we noted an increase of 2.5 -fold for young CRU versus an increase of 3.1-fold for old CRU $(P<0.05)$. Interestingly, CRU of old BM collected and processed in hypoxia were not significantly different from those of young BM collected and processed in ambient air $(P>0.05)$.

Collection and processing of aged mouse BM under hypoxic conditions increases HSC homing to the BM following transplantation. One way to enhance the efficacy of HCT is to increase donor HSC homing to the BM following transplantation (30). Therefore, we assessed the expression density on HSCs and HPCs of CXCR4. CXCR4 is a receptor for chemokine stromal cell-derived factor-1 (SDF-1, also known as CXCL12), implicated by us and others in the migration, homing, and survival of HSCs and HPCs (31-39). Under ambient air conditions, the expression density of CXCR4 was greatly decreased in old versus young LT-HSCs, CMPs, and GMPs and was increased in ST-HSCs, with no significant differences in MPPs or MEPs (Figure 3, A-F). As we previously reported (23), hypoxic collection of young cells demonstrated decreased CXCR4 expression in the LT-HSCs. Here, we report that CXCR4 expres- sion was also decreased in the GMP population but increased in the MEP population of young BM cells, with no differences in expression in ST-HSC, MPP, or CMP populations. In contrast, as we now report, hypoxic collection and processing of $\mathrm{BM}$ from old mice led to increased CXCR4 expression compared with expression levels detected in ambient air-collected/processed old BM LT-HSCs, CMPs, and MEPs, but not ST-HSCs, MPPs, or GMPs. Although expression of CXCR4 is associated with HSC homing capacity, which is an integral part of HSC engraftment capability, CXCR4 expression itself is not an absolute indication of homing and engraftment $(40,41)$. Thus, we performed homing experiments (Figure 3G). Results are expressed as both the percentage of total donor $\left(\mathrm{CD} 45.1^{-} \mathrm{CD} 45.2^{+}\right)$cells (Figure $\left.3 \mathrm{H}\right)$ and absolute numbers of donor $\left(\mathrm{CD} 45.1^{-} \mathrm{CD} 45.2^{+}\right) \mathrm{HSCs}\left(\mathrm{Lin}^{-} \mathrm{Scal}^{+} \mathrm{CKit}^{+}\right.$[LSK] CD150 ${ }^{+}$; Figure 3I). In the acclimated air cohort, old cells homed with lower frequency than did young cells (5.0-fold and 11.2-fold decreases, respectively; Figure 3, H and I) $(5,42)$. However, hypoxiacollected/processed aged cells homed equivalently to acclimated air-collected/processed young total BM cells and HSCs. It 
A
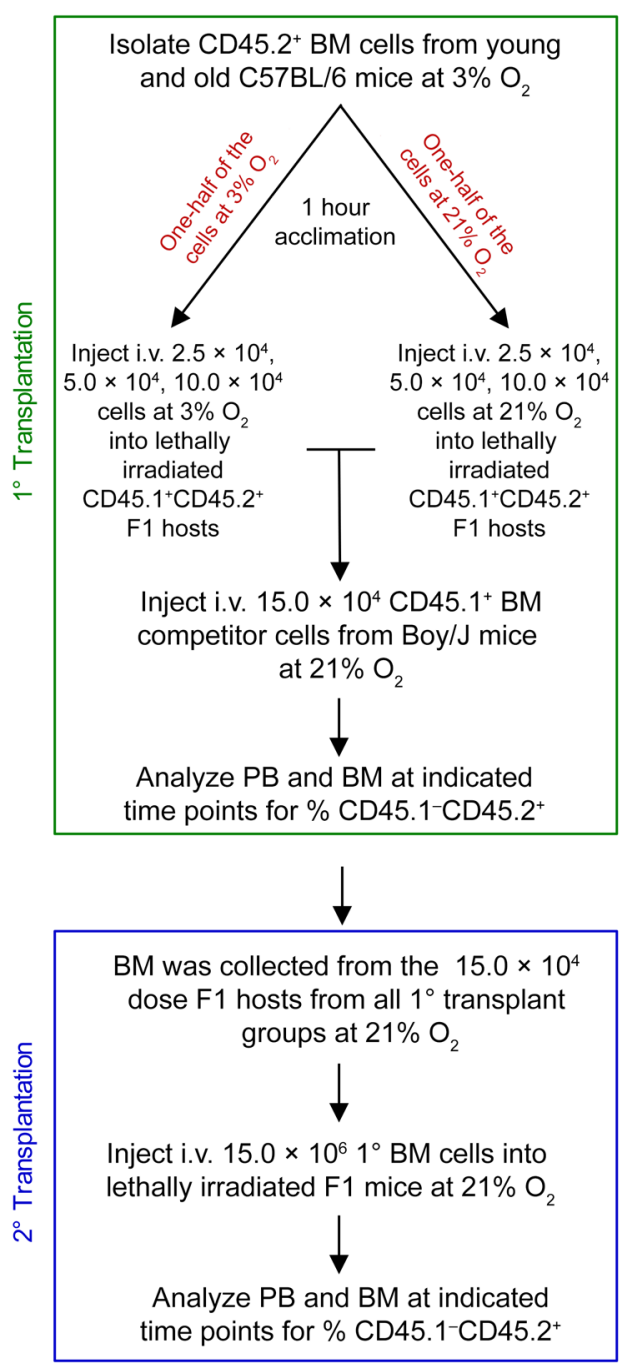

B

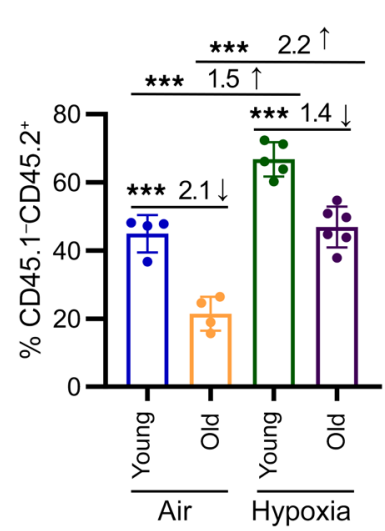

$\mathbf{E}$

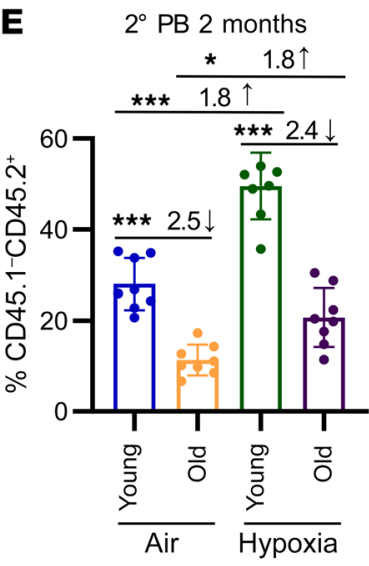

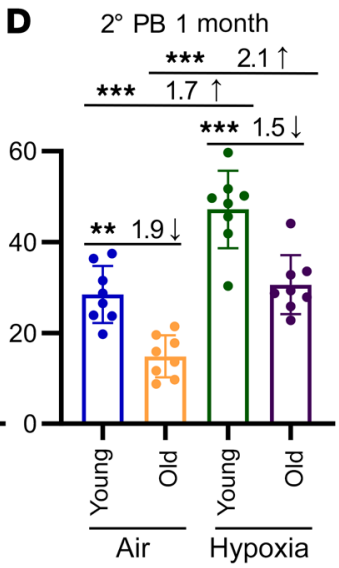

F $\quad 2^{\circ} \mathrm{PB} 4$ months
G $\quad 2^{\circ}$ BM 4 months

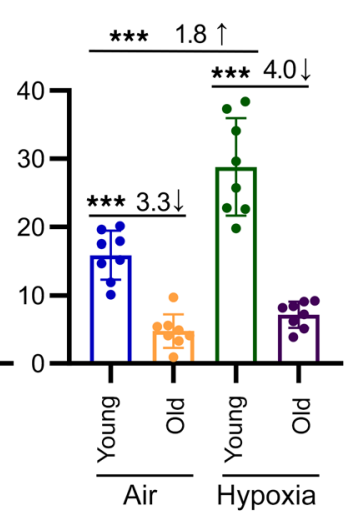

H

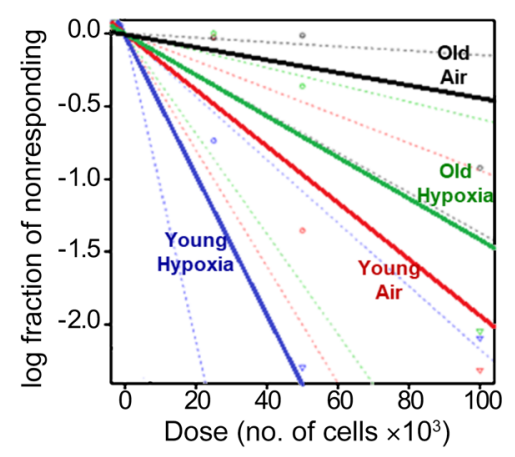

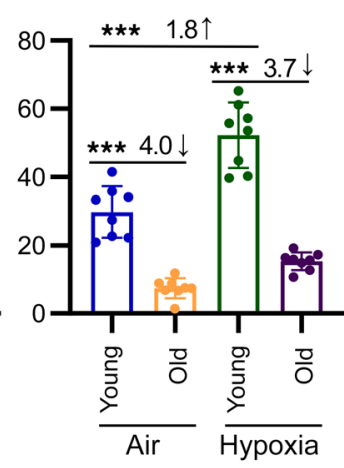

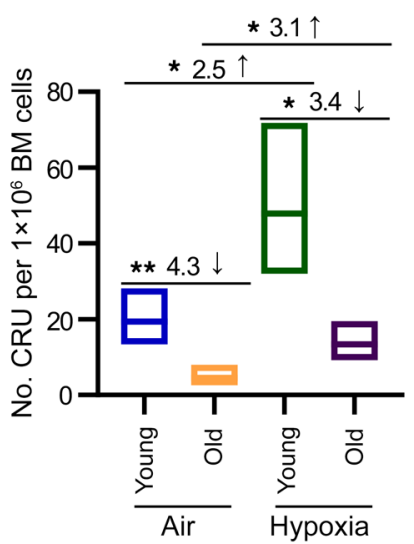

Figure 2. HSC engraftment efficiency of young and old C57BL/6 mice collected/processed under ambient air versus hypoxia as assessed by BM transplantation and limiting dilution analysis. (A) Schematic of the experiment. Donor BM cells collected in ambient air versus hypoxia and competitor cells collected in $21 \% \mathrm{O}_{2}$ were injected i.v. in either $3 \% \mathrm{O}_{2}$ (hypoxia) or $21 \% \mathrm{O}_{2}$ (ambient air) into 950 cGy-irradiated CD45.1 $1^{+} \mathrm{CD} 45.2^{+} \mathrm{F} 1$ recipients (25,000, 50,000, and 100,000 donor cells, with 150,000 competitor cells). The percentages of donor cells (CD45.1-CD45.2+) in PB (B) and BM (C) were determined from the 50,000-cell-dose group. Data represent the mean \pm SEM for 4-6 recipient mice. (D-G) Secondary transplantations of primary recipient BM collected under ambient air conditions. The percentages of donor cells in PB (D-F) and BM (G) were determined. Data represent the mean \pm SEM of 8 mice per group. (H) Left: Poisson statistical analysis from limiting dilution transplantation. Shapes represent the percentages of negative mice for each cell dose, solid lines indicate the best-fit linear model, and dotted lines represent $95 \% \mathrm{Cl}$. Right: Number of CRU in $1 \times 10^{6} \mathrm{BM}$ cells. Data represent the mean \pm SEM for 4-6 F1 recipient mice. (See Supplemental Table 1 for more details.) ${ }^{*} P<0.05$, ${ }^{* *} P<0.01$, and ${ }^{* *} P<0.001$, by 1-way ANOVA with post hoc Tukey's multiple-comparison test (B-G). $1^{\circ}$, primary; $2^{\circ}$, secondary.

is important to note that collection and processing of young BM cells in hypoxia resulted in a significant $(P<0.001)$ reduction in LT-HSC CXCR4 expression, with a trend toward a decrease (not significant) in homing efficiency. This hypoxia-associated reduc- tion in CXCR4 expression and homing of young HSCs did not correlate with the increased engraftment of these cells seen in Figure 2. On the basis of these data and the findings from our limiting dilution analysis from our previously published work (23) (which 
A

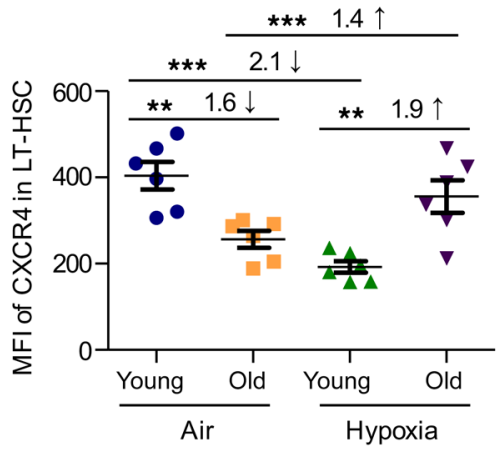

D

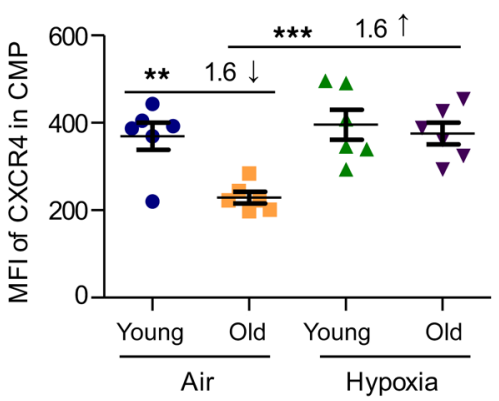

G Isolate $\mathrm{CD} 45.2^{+} \mathrm{BM}$ cells from young and old C57BL/6 mice at $3 \% \mathrm{O}_{2}$

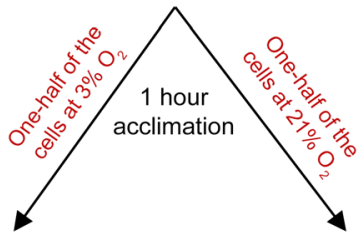

Inject i.v. $12 \times 10^{6}$ cells at $3 \% \mathrm{O}_{2}$ into lethally irradiated

Boy/J mice

$\left(\mathrm{CD} 45.1^{+}\right)$ (CD45.1 $\left.1^{+}\right)$

Inject i.v. $12 \times 10^{6}$ cells at $3 \% \mathrm{O}_{2}$ into lethally irradiated Boy/J mice
B

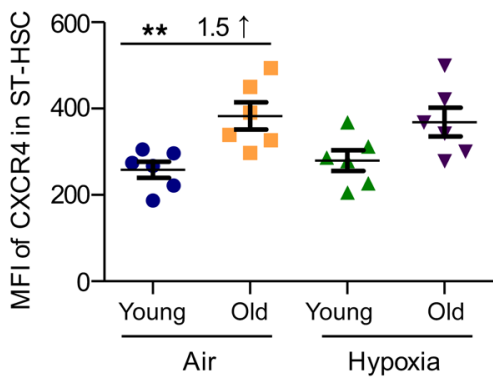

E

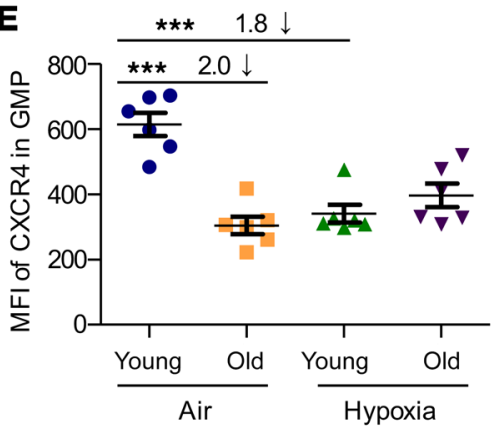

H

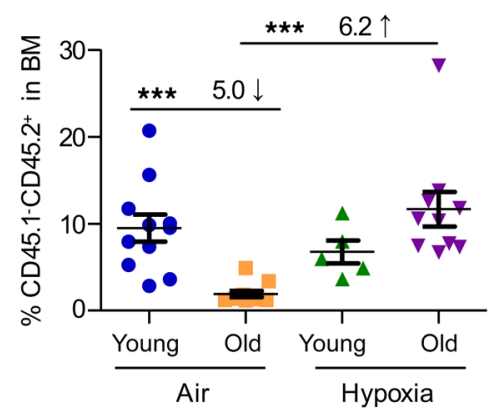

18 hours later isolate BM cells analyze by flow cytometry

J

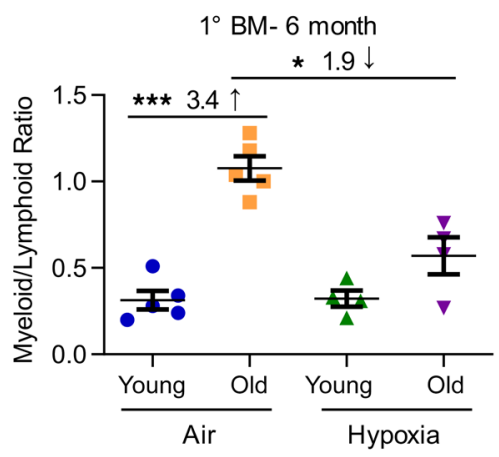

K

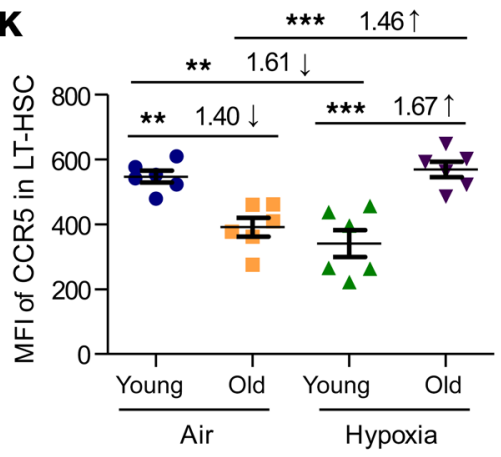

C

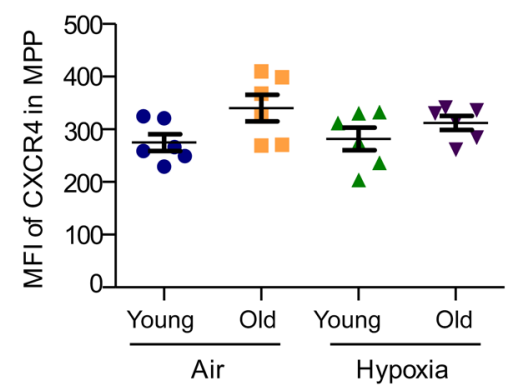

$\mathbf{F}$

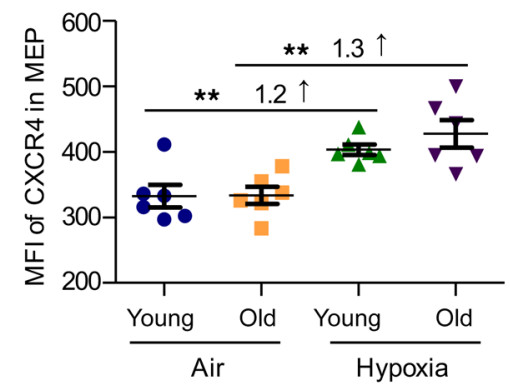

I

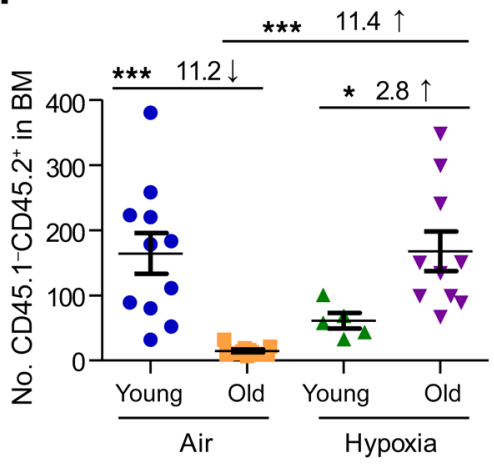

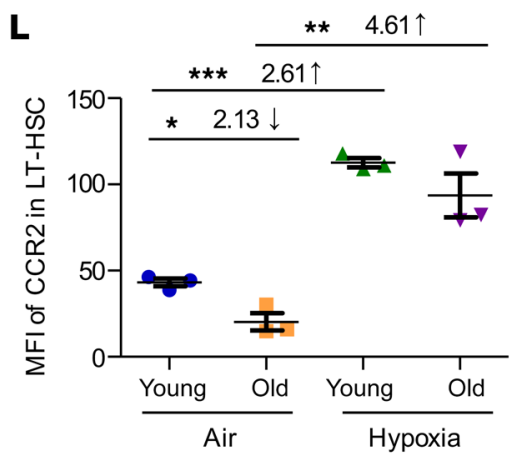

Figure 3. Chemokine receptor expression in young and old C57BL/6 mouse BM collected under hypoxia and processed under ambient air or hypoxic conditions. Young and old C57BL/6 donor BM cells were collected and processed as in Figure 1A. CXCR4 expression on LT-HSCs (A), ST-HSCs (B), MPPs (C), CMPs (D), GMPs (E), and MEPs (F). Data represent the mean \pm SEM for 6 C57BL/6 mice per group. (C) Experimental schema to determine the homing capacity of donor cells. (H) Percentage of CD45.1-CD45.2 $2^{+}$cells in BM determined 18 hours after injection. (I) Number of CD45.1-CD45.2 $2^{+}$(LSK CD150) HSCs

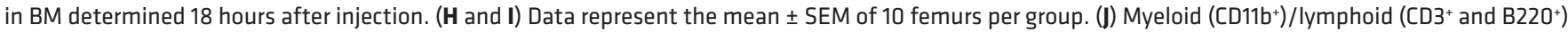
ratio in BM 6 months after primary transplantation. Data represent the mean \pm SEM for 4-6 F1 recipient mice. (K) CCR5 expression on LT-HSCs. Data represent the mean \pm SEM for 6 C57BL/6 mice per group. (L) CCR2 expression on LT-HSCs. Data represent the mean \pm SEM for 3 C57BL/6 mice per group. ${ }^{*} P<0.05,{ }^{* *} P<0.01$, and ${ }^{* * *} P<0.001$, by 1-way ANOVA with post hoc Tukey's multiple-comparison test. 

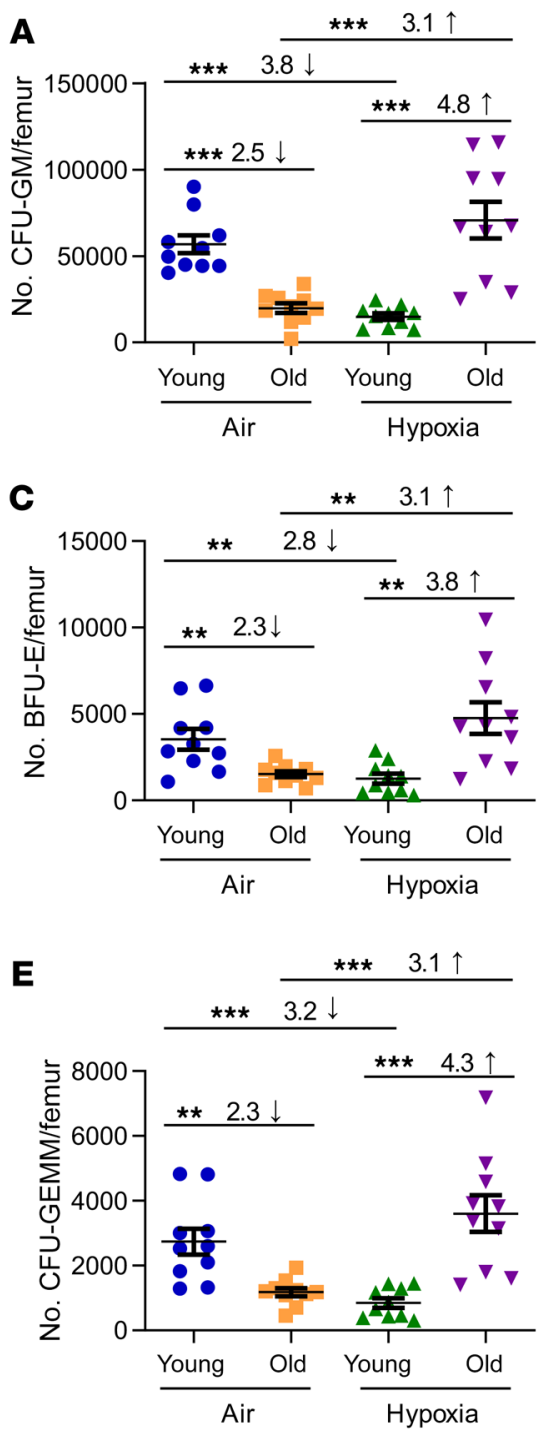
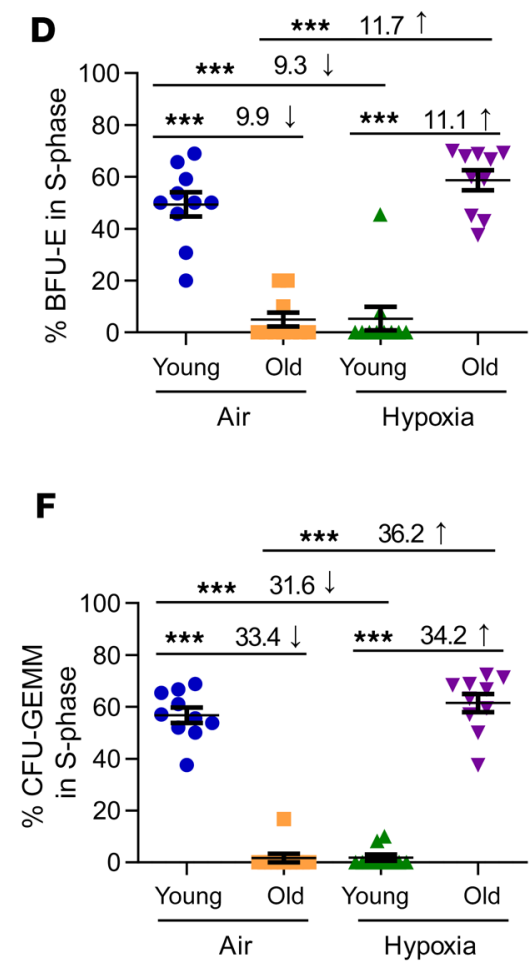

Figure 4. Numbers of functional HPCs from young and old C57BL/6 mouse BM collected under hypoxia and processed under ambient air or hypoxic conditions. BM cells were collected and processed as in Figure $1 \mathrm{~A}$. Nucleated BM cells were used in an HPC CFU assay stimulated in vitro with EPO, SCF, PWMSCM, and hemin $(\mathbf{A}, \mathbf{C}$, and $\mathbf{E})$ and cultured at $5 \% \mathrm{O}_{2}$, with the percentage of HPCs in S-phase defined by a high specific activity tritiated thymidine kill assay (B, $\mathbf{D}$, and $\mathbf{F})$. The numbers of CFU-GM (A and B), BFU-E (C and $\mathbf{D})$, and CFU-GEMM (E and F) were calculated per femur. Data represent the SEM for 10 C57BL/ 6 mice from 3-4 experiments. ${ }^{* *} P<0.01$ and ${ }^{*}{ }^{*} P<0.001$, by 1 -way ANOVA with post hoc Tukey's multiple-comparison test. we replicated in Figure 2), we hypothesize that the enhanced engraftment of hypoxia-isolated/processed BM cells may be due to an increase in the number of functional HSCs in the donor BM cells injected, and not to the number of total HSCs that homed to the BM after transplantation.

Collection and processing of aged mouse BM cells under hypoxic conditions reduces myeloid skewing following transplantation. Old $\mathrm{BM}$ donor cells have abnormal myeloid/lymphoid cell ratios following primary transplantation compared with young BM donor cells, with skewing toward myeloid lineages for old BM donor cells (1-3, 5-9). We also observed this phenomenon for ambient air-acclimated cells (Figure 3J). However, and most important, myeloid/lymphoid cell ratios in recipient mice receiving hypoxiacollected/processed old BM cells were similar to those of younger $\mathrm{BM}$ exposed to ambient air or hypoxia. We detected no change in the myeloid/lymphoid cell ratio for young BM collected and processed in air or hypoxia. This reduction in the myeloid skewness in transplanted hypoxia-collected/processed aged BM correlated with the decreased myeloid progenitor (i.e., CMP and GMP) and increased CLP numbers (by phenotype) observed in old BM in the hypoxia cohort (Figure 1, D-F). These findings suggest that, solely by collecting and processing the aged mouse BM under hypoxic conditions, we were able to alter the functional HSC compartment enough so that we affected the apparent fate decision of these aged, transplanted BM cells.

The RANTES/receptor axis is implicated in the skewing of myeloid/lymphoid cell ratios during aged mouse hematopoiesis (24). CCR5, a receptor for several chemokines including RANTES, MIP-1 $\alpha$, and MIP-1 $\beta$, can form a hetero-oligomer together with CXCR4 and CCR2 to mediate RANTES signaling (24, 43, 44). Therefore, we evaluated CCR5 and CCR2 expression on young and old BM HSCs collected and processed in ambient air versus those collected in hypoxia. We found that expression of CCR5 was modestly but significantly lower in ambient air-acclimated BM LT-HSCs when comparing young and old mice (Figure 3K). Of relevance, CCR5 expression was significantly higher in hypoxiacollected old than in ambient air-acclimated young BM LT-HSCs (Figure 3K). Ambient air-processed young BM cells demonstrated modest decreases in CCR2 expression on LT-HSCs compared with ambient air-processed aged BM cells (Figure 3L). But, interestingly, young and old mouse BM collected and processed under hypoxic conditions demonstrated significant increases in HSC CCR2 
A
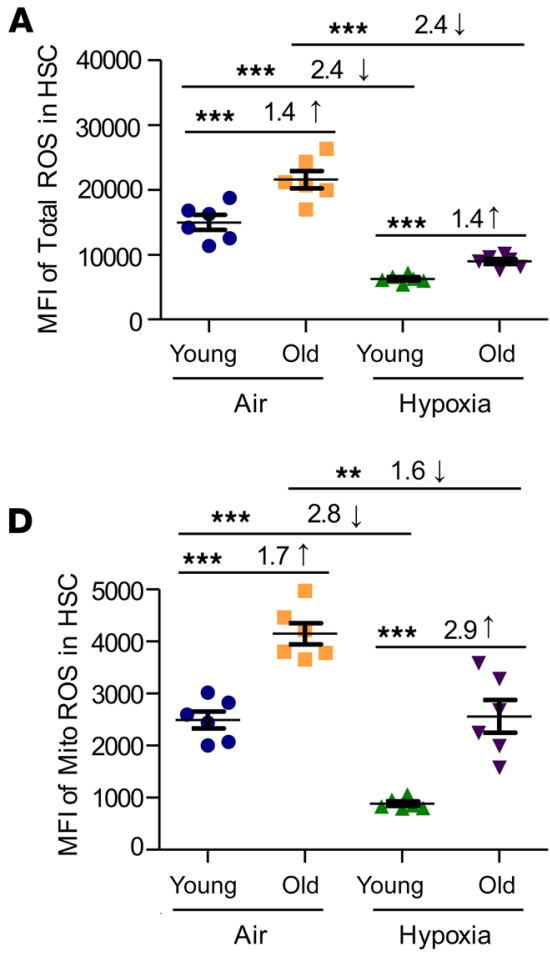

B

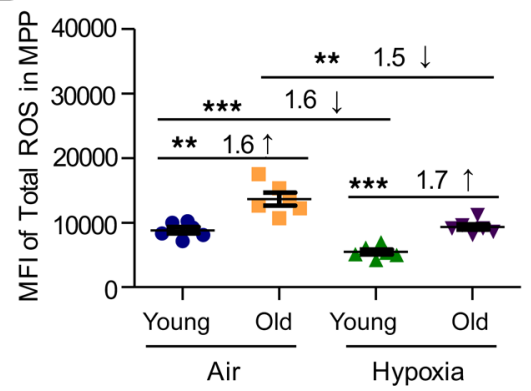

E

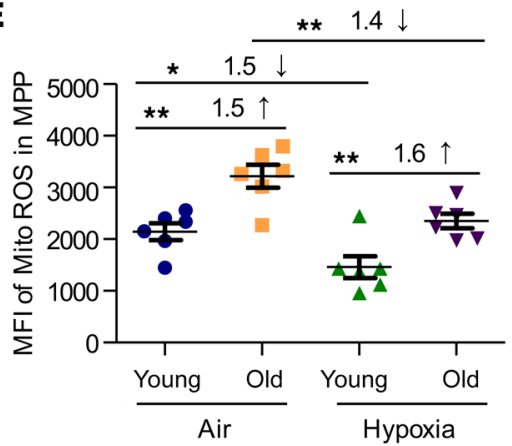

C

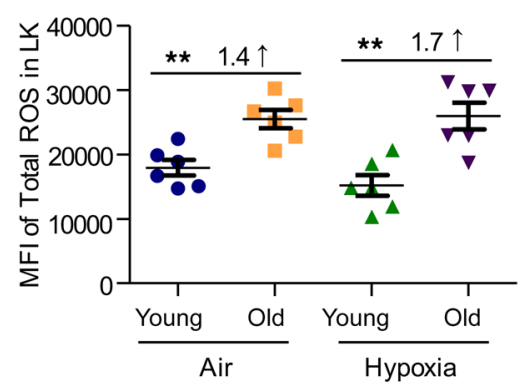

$\mathbf{F}$

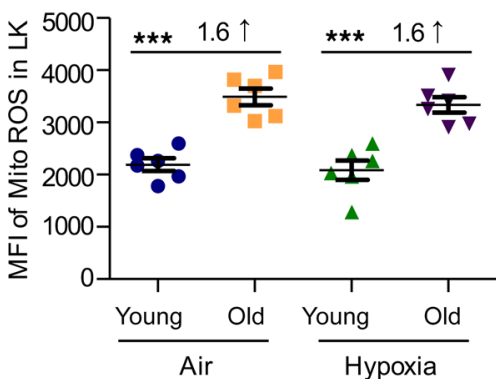

Figure 5. Total and mitochondrial HSC and HPC ROS levels in young and old C57BL/6 mouse BM collected under hypoxia and processed under ambient air or hypoxic conditions. Young and old C57BL/6 mouse BM was collected and processed as in Figure $1 \mathrm{~A}$. BM cells were analyzed by flow cytometry for total ROS levels (using H2DCFDA) (A-C) and mitochondrial (Mito) ROS levels (using MitoTracker Red CMXRos) (D-F). ROS levels in HSCs (LSK CD34-FLT3-) (A and $\mathbf{D})$, MPPs (LSK CD34 ${ }^{+}$Flt3 ${ }^{+}$) (B and E), and LK myeloid progenitors (Lin-Sca-1-c-Kit ${ }^{+}$) (C and F). Data represent the mean \pm SEM for 6 C57BL/6 mice per group. ${ }^{*} P<0.05,{ }^{* *} P<0.01$, and ${ }^{* *} P<0.001$, by 1-way ANOVA with post hoc Tukey's multiple-comparison test.

expression. Thus, "normalization" of myeloid/lymphoid cell ratios for old engrafting BM LT-HSCs was associated with changes in expression of CCR5, CCR2, and CXCR4, all components of RANTES signaling. It is important to note, as we previously reported (23), that collection under hypoxic conditions did not affect the myeloid/lymphoid skew when young BM donor cells were used.

Hypoxic collection and processing of aged BM enhances HPC function. While much is known about HSC characteristics in aging BM following ambient air collection, less is known regarding HPCs. We observed decreased numbers of functional HPCs, as assessed by colony assay, in old versus young BM when the cells were acclimated to ambient air, as previously reported (23). Moreover, old HPCs were in a slow or noncycling state, as assessed by a high specific activity tritiated thymidine kill assay, the only way to assess the cycling of functional HPC populations, compared with HPCs from BM of young mice (Figure 4, C57BL/6 mice, and Supplemental Figure 3, CB6 mice and BALB/c mice). Interestingly, when old BM was collected and processed in hypoxia, we unexpectedly found a significantly higher number of detectable functional HPCs in old BM processed in hypoxia than in old BM processed in ambient air, and these cells were in rapid cell cycle. This demonstrated that young and old BM HPCs manifested differing effects from ex vivo exposure to $3 \% \mathrm{O}_{2}$, with HPCs from old $\mathrm{BM}$ in a rapid cell cycle, whereas young HPCs were in a slow or noncycling state when collections were made in hypoxia. To our knowledge, these effects have not been previously reported and reflect a more accurate in vivo environmental depiction of HPC numbers and cycling status in BM from old mice than that previously reported for ambient air-collected/processed BM.

ROS and heat shock protein levels are changed when old BM is collected and processed under hypoxic conditions. To further explore changes in the numbers and functional activities of HSCs and HPCs from young versus old BM cells, we focused on total and mitochondrial ROS production (Figure 5). Our previous work implicated changes in ROS levels in EPHOSS-induced hematopoietic effects (23). Total ROS (Figure 5, A-C) and mitochondrial ROS (Figure 5, D-F) were significantly elevated in aged, ambient air-acclimated HSC, MPP, and lineage-negative, $\mathrm{Sca1}^{-}, \mathrm{c}^{-\mathrm{Kit}^{+}}$(also known as LK, which contains mainly myeloid HPCs) populations of BM cells compared with ambient air-acclimated young BM. ROS levels were significantly reduced in young HSCs and MPPs collected and processed in hypoxia, as was the case in older cells, although the older cells, even when collected in hypoxia, produced more ROS than did young cells, which means that a decrease in ROS in aged BM HSCs may further increase their functional activities. Within the LK cell population, collection under hypoxia had no effect on total or mitochondrial ROS levels (Figure 5, C and F).

Aging cells are associated with an altered ability to cope with environmental stresses, which can be observed by changes in heat shock protein (HSP) responses (45-48). Many members of the HSP family perform chaperone functions by stabilizing new proteins to ensure correct folding, or by helping to refold proteins damaged by cellular stress $(45,46)$. Upon examination of ambient air-collected/processed young and old mouse BM, we noticed 2 LT-HSC 
A

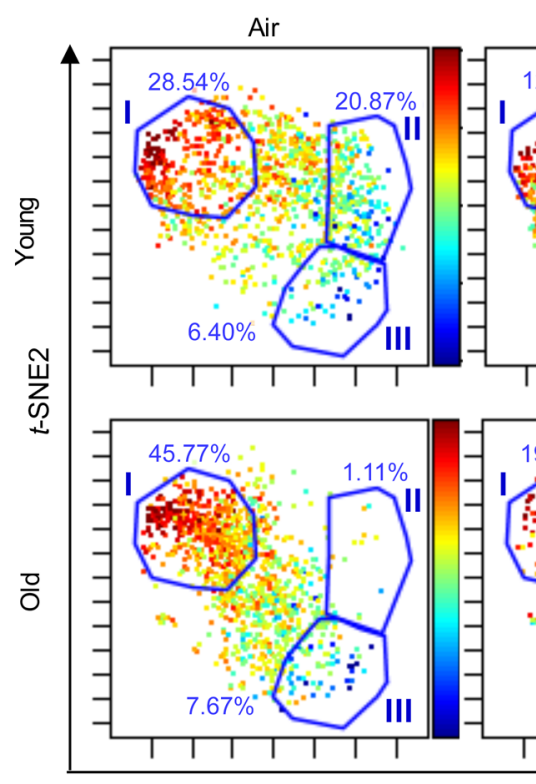

LSK CD48-CD150+ CMyc

Hypoxia

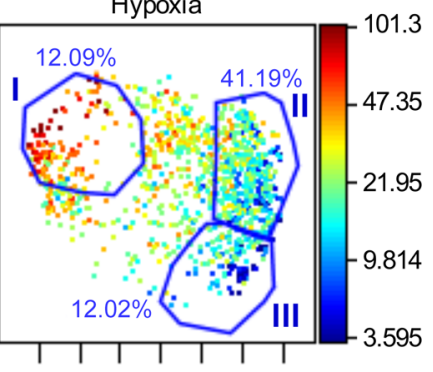

101.3

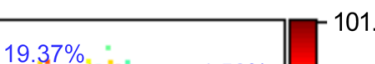

B
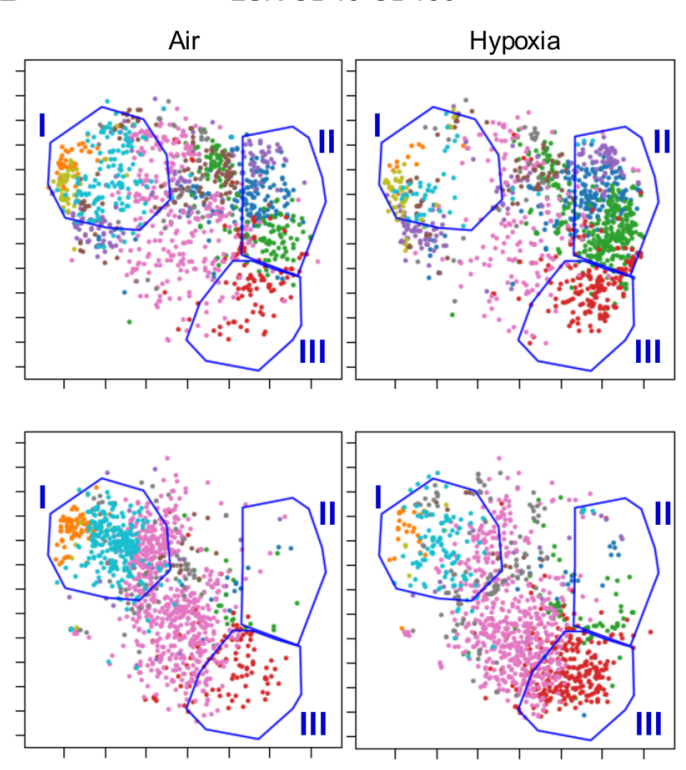

LSK CD48-CD150+

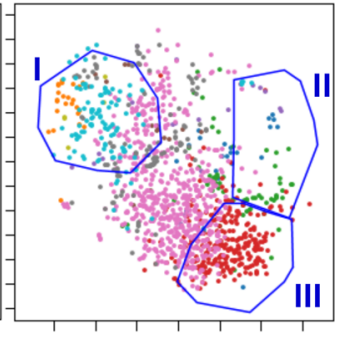

$t$-SNE1

Figure 6. CуTOF analysis of gated LT-HSCs demonstrated distinct subpopulation changes among ambient air-acclimated and hypoxia-collected/processed lineage-negative BM cells. BM from young and old C57BL/ 6 donor mice was collected and processed as in Figure $1 \mathrm{~A}$. Samples were stained with the indicated antibodies conjugated to metal (see Supplemental Table 3) and then analyzed with a CyTOF 2 mass cytometer and Cytobank software. (A) cMyc levels within gated LSK CD48-CD150+ plots using viSNE analysis. Scales indicate the mean marker intensity of arcsinh-transformed values. (B) Gated LSK CD48-CD150+ LT-HSCs were used to make viSNE plots, where different colors indicate the subpopulations explored (10 in all; see Supplemental Table 2 for subpopulation definitions). In A and B, the cell populations changed by ambient air exposure are labeled as gates I, II, and III, and the percentage of cells within the gate is indicated. $t$-SNE1,-2, $t$-distributed stochastic neighbor embedding.

and $\mathrm{LK}$ populations of $\mathrm{HSP}_{4} \mathrm{O}^{+}$cells: low- and high-expressing groups. In young and old mouse BM LT-HSC and LK populations, the percentage of cells expressing high levels of HSP4O in both of these cell populations was greatly reduced when BM was collected and processed under hypoxic conditions (Supplemental Figure 4, A and B). When examining HSP70 expression, we observed a decrease in the percentage of $\mathrm{HSP}^{+} \mathrm{O}^{+}$cells in both LT-HSC and LK populations when the cells were collected and processed under hypoxia (Supplemental Figure 4, C and D). Interestingly, the difference in the percentage of $\mathrm{HSP7O}^{+}$LT-HSCs between the ambient air and hypoxia groups was greater in the BM of aged mice than in that of young mice, suggesting that aged BM LT-HSCs were more susceptible to changes in their HSP70 response to oxidative stress than were young BM LT-HSCs.

Hypoxic collection and processing of both young and old BM cells results in decreased $c M y c$, pmTOR, and PI3K levels within distinct subpopulations of LT-HSCs. To determine whether exposure of LT-HSCs from young and aged mouse BM to ambient air results in alterations in the expression of several important factors that influence HSC function, we performed cytometry by time-offlight (CyTOF) analysis of BM isolated and processed in ambient air or hypoxia and specifically examined the population of $\mathrm{BM}$ LT-HSCs (LSK CD48-CD150 ${ }^{+}$; Figure 6, Supplemental Figures 5 and 6, and Supplemental Table 2). Expression of CD166, Oct3/4, TNF- $\alpha$, PDGF $\beta$, IGF1, TGF- $\beta$, C/EBP $\beta$, BMP4, Runx1, phosphorylated AKT (p-AKT), p-STAT3, cMyc, Notch, Gfi1, PI3K, p-ERK, and pmTOR (see Supplemental Table 3 for the full names of the proteins) was examined using visualization of $t$-distributed stochas- tic neighbor embedding (viSNE) analysis of normalized CyTOF events. cMyc, PI3K, and mTOR all play an important role in the regulation of normal and malignant hematopoiesis by regulating HSC, HPC, and leukemia-initiating stem cell metabolism, growth, proliferation, and survival, and are important for the correct balance between self-renewal and differentiation of HSCs (49-51). These 3 markers (cMyc, PI3K, and pmTOR) in our CyTOF analysis were consistently changed between ambient air and hypoxia collections when examining young and old BM LT-HSC populations; therefore, we examine the expression levels of these proteins in individual cells within the LT-HSC population. The high $\mathrm{cMyc}^{-}$ expressing LT-HSC populations (gate I: $25.54 \%$ in young, ambient air LT-HSCs, $12.09 \%$ in young, hypoxia LT-HSCs, $45.77 \%$ in old, ambient air LT-HSCs, and $19.37 \%$ in old, hypoxia LT-HSCs; Figure 6A) in both young and old mice were reduced, and the lower cMyc-expressing LT-HSC populations were increased (gate II: $20.87 \%$ in young, ambient air LT-HSCs, $41.19 \%$ in young, hypoxia LT-HSCs, $1.11 \%$ in old, ambient air LT-HSCs, and $4.58 \%$ in old, hypoxia LT-HSCs; gate III: $6.40 \%$ in young, ambient air LT-HSCs, $12.02 \%$ in young, hypoxia LT-HSCs, $7.67 \%$ in old, ambient air LT-HSCs, and $26.17 \%$ old, hypoxia LT-HSCs) when BM cells were isolated and processed under hypoxic conditions. We identified the markers associated with these indicated gated regions (Figure $6 \mathrm{~B}$ ). For the younger mice, we observed an increase in what we identified as LT-HSC subpopulation 2 (within gate I, orange dots; see Supplemental Table 2 for subpopulation definitions) and subpopulation 10 (within gate I, teal dots) when cells were collected and processed under ambient air conditions. Subpopulation 
2 was determined to be a CD150 ${ }^{\text {hi }} \mathrm{cMyc}$ hi IGF $1^{+}$Runx $1^{+} \mathrm{PDGF} \beta^{\mathrm{hi}}$ Gfi1 ${ }^{\text {hi }}$-Erk $1 / 2^{+}$TGF- $\beta^{\text {hi }}{ }^{\text {TNF }}-\alpha^{+}$BMP $4{ }^{\text {hi }}$ p-Akt ${ }^{+}$PI $3 K^{\text {hi }}{ }^{\text {pmTOR }}{ }^{\text {hi }}$ $\mathrm{C} / \mathrm{EBP} \beta^{\text {hi }}$ LT-HSC population. Subpopulation 10 was determined to be a CD150 ${ }^{\text {hi }}$ CMych $^{\text {hi } I G F 1+R u n x} 1^{+}$PDGF $\beta^{+}$Gfi $1^{\text {hi }}$ p-Erk1 $1 / 2^{+}$TGF- $\beta^{+}$ $\mathrm{BMP}^{+} \mathrm{p}-\mathrm{Akt}^{+} \mathrm{PI} 3 \mathrm{~K}^{\mathrm{hi}} \mathrm{pmTOR}{ }^{\mathrm{hi}} \mathrm{C} / \mathrm{EBP} \beta^{\text {hi }}$ LT-HSC population. For the aged mice, we noted an increase in what we identified as LT-HSC subpopulation 4 (within gate III, red dots,) which was discovered to be a $\mathrm{CD} 150^{+} \mathrm{CMyc}+\mathrm{IGF} 1^{\text {lo }} \mathrm{Gfii} 1^{+} \mathrm{TGF}-\beta^{\text {lo }} \mathrm{BMP} 4^{+} \mathrm{PI} 3 \mathrm{~K}^{+}$ pmTOR $^{+}$LT-HSC population when isolated under hypoxic conditions. For young BM LT-HSCs, isolation and processing of cells under hypoxic conditions resulted in an increase in LT-HSC subpopulation 3 (within gate II, green dots), defined as a CD150 $\mathrm{cMyc}^{+} \mathrm{IGF} 1^{\text {lo }}{ }^{\text {Runx }} 1^{+} \mathrm{Gfi1}^{+}{ }^{+} \mathrm{TGF}-\beta^{\text {lo }} \mathrm{BMP}^{+}{ }^{+} \mathrm{PI} 3 \mathrm{~K}^{+} \mathrm{pmTOR}^{+} \quad$ LT-HSC population. When examining pmTOR and PI3K expression in our LT-HSC populations (Supplemental Figure 5), we observed shifts between ambient air and hypoxia in young and old mice that were identical to those seen with cMyc. This suggests that exposure to ambient air results in increased numbers of phenotypically defined LT-HSCs within subpopulations of LT-HSCs (subpopulations 2 and 10) that express higher levels of cMyc, pmTOR, and $\mathrm{PI} 3 \mathrm{~K}$, all factors involved in the regulation of HSC maintenance and the balance between normal HSCs and malignant leukemiainitiated stem cell transformation (49-51).

\section{Discussion}

Following transplantation of mouse $\mathrm{BM}$ isolated and processed under hypoxia, we observed a significant enhancement of the engraftment potential of aged BM that was associated with an increase in HSC function efficient enough to make the aged donor $\mathrm{BM}$ cells equivalent to young donor BM cells processed under ambient air conditions. Thus, although the hypoxia-collected/processed aged BM HSCs were not as efficient as hypoxia-collected/ processed young BM HSCs, these findings open up the possibility that aged BM cells may be effective donor cells for use in clinical autologous and allogeneic HCT. We still observed a significant increase in the number of phenotypically defined LT-HSCs (2.0fold increase) in the $\mathrm{BM}$ of aged mice prior to transplantation compared with $\mathrm{BM}$ of young mice when both were processed and maintained under hypoxia. This observation suggests that hypoxic collection and processing of aged BM cells does not fully revert the aging phenotype (i.e., increased HSC numbers associated with aging) within the BM. However, our transplantation results demonstrate that there was at least a portion of these HSCs that were more functional. As of yet, we are unsure about which HSC subpopulation was responsible for this increased engraftment efficiency.

Of interest, our CyTOF analysis revealed potential subpopulations of LT-HSCs that may be responsible for the effects of hypoxia on enhancement of HSC function. For young mice, the CD150 $\mathrm{cMyc}^{+} \mathrm{IGF} 1^{\text {lo }}$ Runx $1^{+} \mathrm{Gfi}^{+}{ }^{+} \mathrm{TGF}-\beta^{\mathrm{lo}} \mathrm{BMP} 4^{+} \mathrm{PI} 3 \mathrm{~K}^{+} \mathrm{pmTOR}^{+}$LT-HSC population (defined as subpopulation 3; see Figure 6 and Supplemental Table 2) was increased in hypoxia-collected BM cells. For aged mice, the $\mathrm{CD} 15 \mathrm{O}^{+} \mathrm{cMyc}^{+} \mathrm{IGF}{ }^{1 \circ} \mathrm{Gfi} 1^{+} \mathrm{TGF}-\beta^{\text {lo }} \mathrm{BMP} 4^{+} \mathrm{PI} 3 \mathrm{~K}^{+} \mathrm{pmTOR}^{+}$ LT-HSC population (defined as subpopulation 4) was increased in hypoxia-collected BM cells. Both of the LT-HSC subpopulations that increased following hypoxic collection were found to be low expressers of IGF and TGF- $\beta$. It has been reported previously that deficiency in IGF signaling promotes hematopoietic recovery following chemo- toxicity and is able to reverse age-dependent myeloid bias (52). It has been shown that, when in the presence of TGF- $\beta$, cycling HSCs are not able to return to quiescence, suggesting that TGF- $\beta$ is a negative regulator of HSC numbers and reconstitution activity once HSCs have entered the cell cycle (53). In addition, we observed a subsequent reduction in the cMyc-, pmTOR-, and PI3K-high-expressing LT-HSC subpopulation within the aged BM following hypoxic isolation and processing of these cells. In previous studies involving young mice, enforced cMyc expression led to a loss of HSC selfrenewal capacity associated with an increase in HPC proliferation and differentiation (49). Various models have shown that activation of the PI3K/mTOR pathways resulted in HSC exhaustion due to hyperproliferation that resulted in a poor repopulation potential of these cells $(50,51,54)$. Since the LT-HSC subpopulations with reduced cMyc, pmTOR, and PI3K expression were enhanced when young and old BM were isolated under hypoxic conditions and these population were also low in both IGF and TGF- $\beta$, it is possible that we were preserving a population of cells that were primed for better and balanced regenerative capability following transplantation. Taken together, our data suggest that isolation of BM under hypoxia preserves the subpopulations most likely to have increased engraftment capability.

It has been well established that oxidative stress plays an important role in stem cell aging. Excessive production of ROS by stem cells compromises cellular repair mechanisms and metabolic homeostasis that ultimately affect the ability of stem cells to self-renew and eventually become functionally depleted (55). It has previously been shown that ROS ${ }^{\text {hi }}$ HSC-enriched cells had significantly less engraftment capability than did ROS ${ }^{\text {lo }}$ HSCenriched cells in serial transplantation experiments. ROS ${ }^{\text {hi }}$ HSCenriched cells have high levels of activated mTOR (54). Inhibition of mTOR with rapamycin or reduction of ROS levels using antioxidants such as $\mathrm{N}$-acetyl-cysteine (NAC) can increase the number of functional HSCs (56). We demonstrate here that HSCs (both from young and old mice) isolated and processed under hypoxia had lower levels of ROS (Figure 5) and lower percentages of pmTOR ${ }^{\text {hi }}$ LT-HSCs (Supplemental Figure 5A), suggesting that LT-HSC populations identified by CyTOF as having lower levels of pmTOR following collection and processing under hypoxia may be the population of LT-HSCs that are more functional. However, further experimentation is required to make this conclusion. Furthermore, our laboratory has previously published a study (57) showing that combinations of different antioxidants (NAC and ascorbic acid 2-phosphate [AAP]) in the media used for isolation and processing of mouse BM cells results in levels of preserved HSC numbers similar to those seen in hypoxia-isolated/processed BM cells. We have also recently reported that using cold temperature $\left(4^{\circ} \mathrm{C}\right)$ during the isolation and processing of human cord blood cells preserves HSC numbers and function (58). These data suggest that use of a combination antioxidant therapy (demonstrated using mouse $\mathrm{BM}$ ) and/or cold therapy (demonstrated using human cord blood) while isolating donor cells may be a potential alternative approach to the use of hypoxia isolation and processing to enhance the efficacy of aged HSCs, although further experimentation is required.

A most interesting finding is that isolation and processing of aged BM in hypoxia resulted in a reversal of the myeloid bias associated with aging following transplantation (see Figure 3J). This 
finding was supported by the fact that, when we phenotypically analyzed the BM from aged mice prior to use in transplantation, we noted an increase in CLPs and a decrease in CMPs and GMPs when the cells were isolated processed under hypoxia (see Figure 1). However, older mice still show a disordered myeloid/lymphoid cell ratio in their blood when their HSCs and HPCs reside in their natural hypoxic environment (i.e., when examining aged donor mice) (1, 3, 5-9). Since HSCs collected under hypoxia demonstrated a normalized myeloid/lymphoid cell ratio after transplantation, it may imply that the BM microenvironment in older mice strongly influenced the bias toward myeloid cell output. The aged BM niche itself is associated with increased ROS levels, which are known to induce altered aged mesenchymal stem cell differentiation and thus an increase in adipocytes $(2,24,59)$. The increased adiposity (associated with a subsequent reduction in osteogenesis) results in changes in terms of which extrinsic factors are secreted (e.g., RANTES) and how much of these factors (e.g., SDF-1) are secreted by cells in the BM HSC niche $(2,24)$. Thus, changes in chemokines/cytokines, growth factors, and even hormones can skew HSCs toward myeloid instead of lymphoid differentiation (2). We observed that hypoxic isolation and processing of aged BM HSCs resulted in changes in the expression of several chemokine receptors that respond to these niche signals (i.e., CXCR4, CCR2, and CCR5). It is possible that, after transplantation, these cells with altered receptor expression were placed into a young mouse BM niche and thus receiving different systemic and niche signals that altered their ability to skew myeloid or lymphoid as well. Regardless, we succeeded in the main goal of our work, which was to determine whether we could collect better engrafting HSCs with normal myeloid skewing from aged $\mathrm{BM}$ and then derive a more potent engrafting HSC population. This implicates the potential use of hypoxia-collected/processed aged HSCs, or other means to achieve this goal, for improved donor cell engraftment.

Of note, it has been reported that the aged BM microenvironment may be less hypoxic than that of young $\operatorname{BM}(2,60)$. Although it is not yet mechanistically clear how much of an effect this may have on the differentiation and self-renewal capacity of HSCs, it may suggest that the HSCs in aged BM are already undergoing or are prone to EPHOSS-induced changes, and that simply isolating and maintaining these cells under hypoxic conditions prior to transplantation sufficiently lessens their "stress" to preserve HSC function. Overall, the reduction in both total and mitochondrial ROS production in HSCs and the decrease in HSP40/HSP70-high populations of HSCs and HPCs would indicate that the isolation and processing of BM under hypoxia keeps the cells in a less stressed state, thereby enhancing the efficacy of both young and old BM cells (especially that of old BM HSCs) for use as donor cells in HCT.

Hypoxic collection and processing of $\mathrm{BM}$ may be a means to enhance the efficacy and use of aged BM HSCs and HPCs as donor cells for HCT. However, to fully translate our work in animal studies to humans in the clinic, alternate approaches might need to be considered. Our laboratory has previously published that the use of a combination of different antioxidants (NAC and $\mathrm{AAP}$ ) in the media used for isolation and processing of young BM cells results in levels of preservation of HSC numbers similar to those seen in hypoxia-isolated/processed young BM cells (57). Although this has not yet been tested in the collection and process- ing of aged mouse BM or human BM donor cells, it is an attractive possibility, as is the collection and processing of aged cells at cold temperatures $\left(4^{\circ} \mathrm{C}\right)(58)$. In addition, we have recently published findings showing that mobilized mouse PB (using G-CSF alone or G-CSF in combination with AMD3100 and plerixafor) collected and processed at lowered oxygen tensions yields enhanced numbers of functional HSCs (61). The collection of blood under hypoxic conditions may be a more obtainable goal than the collection of BM from patients or human donors. But no matter whether we develop a technique to mimic collection under hypoxic conditions or collect donor HSCs from an alternative source than the BM, in this work, we have shown that by isolating and collecting BM in an environment that more closely mimics that of in vivo lowered $\mathrm{O}_{2}$ tension conditions, we could reduce the "stress" these cells were undergoing during the collection process. We suggest that collection of HSCs from older individuals under hypoxic conditions may be more useful for clinical HCT to treat malignant and nonmalignant disorders. Isolation of HSCs and HPCs under hypoxic conditions also resulted in a decrease in subpopulations of HSCs that were high expressers of cMyc, pmTOR, and PI3K levels, all of which are known, when dysregulated in HSCs, to induce hematological malignancies (49-51). This finding might indicate a use for hypoxic collection of aged BM to generate HSCs that are less likely to progress to a malignant state upon transplantation.

\section{Methods}

Mice. Young (8-12 weeks of age) and old (20-28 weeks of age) C57BL/6, BALB/c, and CB6F1 (BALB/c C57BL/6 F1) male and female mice were obtained from the NIH's National Institute of Aging Rodent Ordering System or from The Jackson Laboratory. Male and female C57BL/6, Boy/J, and B6 Boy/J F1 (8-10 weeks of age) were obtained from an on-site breeding core facility at the Indiana University School of Medicine. Animals were maintained under temperature- and lightcontrolled conditions $\left(21^{\circ}-24^{\circ} \mathrm{C}, 12\right.$-hour light/12-hour dark cycle) and were group housed according to age and sex. Mice were fed ad libitum. For all experiments, mice were matched by sex, and the groups contained both male and female mice.

$B M$ cell harvest. Once mice were euthanized, the animals were immediately passed through an air lock chamber and then into a temperature-, humidity-, $\mathrm{O}_{2}^{-}$, and $\mathrm{CO}_{2}$ - controlled, custom-built glove box (Hypoxic Chamber, Coy Lab Products) (23). The glove box was maintained at $3 \% \mathrm{O}_{2}$ by $\mathrm{N}_{2}$ balance and $5 \% \mathrm{CO}_{2}$. Femurs were collected and flushed in sterile PBS (HyClone, GE Healthcare Life Sciences) within the glove box. Collected BM cells were then split in half, with one half kept in the hypoxic glove box and the other half moved to a biosafety hood exposed to ambient air conditions $\left(21 \% \mathrm{O}_{2}\right)$, where they remained for 1 hour prior to further processing so that the BM cells could acclimate to ambient air conditions, as we described previously (23). Subsequent procedures such as staining cells for flow cytometry or setting up colony assays were performed simultaneously in the hypoxic glove box or in the biosafety cabinet under ambient air conditions (see Figure 1A for the experimental design). When culturing cells under hypoxic conditions, the cells were equilibrated in the hypoxic glove box, such that at no point were the cells exposed to ambient air conditions, and the cells were then transferred from the glove box to an incubator maintained at $5 \% \mathrm{O}_{2}$ by $\mathrm{N}_{2}$ balance and $5 \% \mathrm{CO}_{2}$ via airtight containers. All solutions (e.g., fixatives), media, reagents (e.g., 
antibodies), plasticware, pipette tips, sterile instruments, and gauze, as well as anything else that could come into contact with the femurs and cells (including the 70\% ethanol used to sterilize the mouse prior to removal of the femur) used for collection and processing under hypoxia were pre-equilibrated to hypoxic conditions in the $3 \% \mathrm{O}_{2}$ glove box for at least 18 hours prior to use. All liquids (especially the methylcellulose used for colony assays because of its viscosity) were vortexed vigorously to displace as much oxygenated air as possible. We emphasize the absolute requirement for extensive equilibration of all materials to the hypoxic conditions of the glove box prior to use and rigorous attention to details to obtain stable and reproducible results for the hypoxia-collected/processed cells. The reagents used for ambient air-acclimated cells were kept under ambient air conditions.

Flow cytometry. For analysis of HSC and HPC cell phenotypes in murine BM, cells were collected at a concentration of approximately $2 \times 10^{6}$ to $3 \times 10^{6}$ cells per tube, washed in PBS, incubated in a fluorescence-conjugated anti-mouse antibody cocktail for 20 minutes at room temperature (RT), washed again in PBS, and then fixed in 1\% formaldehyde. Samples were analyzed on an LSR II Flow Cytometer (BD Biosciences). Single-color compensation and isotype controls were included for each experiment. Data analysis was performed using FlowJo 7.6.3 software (Tree Star). Gates were determined using fluorescence-minus-one controls. The percentage of each cell population was used to calculate the absolute numbers of each cell population per femur. The following phenotyping markers were used: FITC- or Pacific Blue mouse lineage cocktail (CD3, Gr-1, CD11b, CD45R, Ter119; BioLegend; catalogs 133302 and 133310), PE-CF594-anti-Ly6A/E (also known as Sca-1; clone D7; BD Biosciences), APC-H7-anti-CD117 (also known as c-Kit; clone 2B8; BD Biosciences), APC- or PE-anti-CD135 (also known as Flt3; clone A2F10.1; BD Biosciences), PE- or BV421anti-CD34 (clone RAM34; BD Biosciences), PerCP-Cy5.5-anti-CD16/ CD32 (Fc $\gamma$ R; clone 2.4G2; BD Biosciences), BV421-anti-CD127 (also known as IL-7R; clone SB/199; BD Biosciences), PE- or BV421-antiCD150 (Q38-480; BD Biosciences), FITC-or BV421-anti-CD48 (clone HM48-1; BD Biosciences), and FITC- or BV421-anti-CD41 (clone MWReg30; BD Biosciences). HSC and HPC populations for mice were defined as follows: LSK cells: Lin $^{-} \mathrm{Sca}-1^{+} \mathrm{C}-\mathrm{Kit}^{+}$; LT-HSCs: LSK Flt3 CD34- or LSK CD150+CD48-CD41-; ST-HSCs: LSK Flt3-CD34+' ${ }^{+}$MPPs: LSK Flt $3^{+} \mathrm{CD}^{2} 4^{+}$; CMPs: Lin ${ }^{-} \mathrm{Sca}-1^{-}{ }^{-}-\mathrm{Kit}^{+} \mathrm{Fc} \gamma \mathrm{II} / \mathrm{IIIR}^{\text {lo }} \mathrm{CD}^{2} 4^{+}$; GMPs: Lin ${ }^{-}$ Sca-1 ${ }^{-}$c-Kit ${ }^{+} \mathrm{c} \gamma \mathrm{II} / \mathrm{IIIR}^{\mathrm{hi}} \mathrm{CD} 34^{+}$; MEPs: Lin $^{-} \mathrm{Sca}-1^{-}{ }^{-}-\mathrm{Kit}^{+} \mathrm{Fc} \gamma \mathrm{II} / \mathrm{IIIR}^{-} \mathrm{CD} 34^{-}$; and CLPs: Lin ${ }^{-} \mathrm{Sca}-1^{10} \mathrm{c}-\mathrm{Kit}^{10} \mathrm{Flt} 3^{+} \mathrm{IL}-7 \mathrm{R}^{+}$. For analysis of transplantation and homing experiments, APC-anti-CD45.2 (clone 104), FITC-antiCD45.1 (clone A20), PerCP-Cy 5.5-anti-CD45R/B220 (clone RA36B2), PE-Cy 7-anti-CD11b (clone M1/70), and BV421-anti-CD3 $\varepsilon$ (clone 145-2C11) were used (all from BD Biosciences). To examine chemokine receptor levels, APC-anti-CD184 (CXCR4; clone 2B11/ CXCR4; BD Biosciences), Alexa Fluor 647-anti-CD195 (CCR5; clone HM-CCR5; BioLegend), and Alexa Fluor 647-anti-CD192 (CCR2; clone SA203G11; BioLegend) were used in a procedure similar to that described above. To assess mitochondrial and total ROS levels, we used CM-H2DCFDA (C6827; Invitrogen, Thermo Fisher Scientific) and MitoTracker Orange CM-H2TMRos (M7511; Invitrogen, Thermo Fisher Scientific) according to the manufacturer's instructions. To examine HSP70 and HSP40 expression levels, anti-HSP40 (ab69402; Abcam), anti-HSP70 (ab181606; Abcam), Alexa Fluor 647 F(ab')2 donkey anti-rat IgG fragment (1:300; Jackson ImmunoResearch), and Alexa Fluor $647 \mathrm{~F}\left(\mathrm{ab}^{\prime}\right) 2$ goat anti-rabbit IgG fragment (1:300; Jackson
ImmunoResearch) were used. For all antibodies used in these studies, the validation for the relevant species and applications can be found on the indicated manufacturer's website.

Engraftment studies and limiting dilution analysis. Competitive transplantations were performed using donor BM cells $(2.5 \times$ $10^{4}, 5 \times 10^{4}$, or $1 \times 10^{5}$ ) from young and old C57BL/6 mice (CD45.1CD $45.2^{+}$) isolated as described in Figure 2A (23). In order to protect hypoxia-isolated donor cells from oxidative stress during injection, we injected hypoxia-isolated donor cells i.v. into B6 Boy/J F1 mice $\left(\mathrm{CD} 45.1^{+} \mathrm{CD} 45.2^{+}\right)$that had been lethally irradiated (950 cGy) 24 hours prior to transplantation and placed within the hypoxic glove box prior to i.v. injection. To achieve this, we used a mouse respirator/injector inside the hypoxic glove box (23). Mice were placed into an ambient air-filled, airtight container and passed through the airlock system of our glove box. The animal was then very rapidly placed into the respirator that pumped normal ambient air to the mouse so that only the tail was exposed to hypoxic conditions. The animal was allowed to acclimate for several minutes prior to injection. After injection, the airflow was turned off, and the mouse was removed from the chamber in reverse of the above procedure. Ambient air-acclimated cells were injected under ambient air conditions. Finally, in a separate injection, $1.5 \times 10^{5}$ competitor $\mathrm{BM}$ cells isolated under ambient air conditions from Boy/J mice $\left(\mathrm{CD} 45.1^{+} \mathrm{CD} 45.2^{-}\right)$were injected into all recipient mice under ambient air conditions (see experimental outline in Figure 2A). At the indicated time points, the percentages of donor CD 45.1 $1^{-} \mathrm{CD} 45.2^{+}$cells in $\mathrm{PB}$ and $\mathrm{BM}$ were determined by flow cytometry. The myeloid/lymphoid $\left(\mathrm{CD} 11 \mathrm{~b}^{+} / \mathrm{CD} 3 \mathrm{e}^{+} \mathrm{B} 22 \mathrm{O}^{+}\right)$ratio in donor cells $\left(\mathrm{CD} 45.1^{-} \mathrm{CD} 45.2^{+}\right)$in the $\mathrm{BM}$ of engrafted mice was determined by flow cytometry at the 6-month point. For limiting dilution analysis, BM engraftment at 6 months was performed as previously described (62), and CRU frequency was calculated using L-Calc software (STEMCELL Technologies) and plotted using extreme limiting dilution analysis (ELDA) software (http://bioinf.wehi.edu.au/ software/elda/). For secondary transplants, $1.5 \times 10^{6} \mathrm{BM}$ cells from the above primary recipient mice were i.v. injected into lethally irradiated B6 Boy/J F1 mice in ambient air. The percentages of CD 45.1 ${ }^{-} \mathrm{CD} 45.2^{+}$ in $\mathrm{PB}$ and $\mathrm{BM}$ were evaluated at the indicated time points (see Figure 2A for the experimental schema).

HPC colony and tritiated thymidine kill assays. For HPC colony assays, BM cells flushed from femurs of the indicated mice were plated at $5 \times 10^{4}$ cells $/ \mathrm{mL}$ in $1 \%$ methylcellulose culture medium with $0.1 \mathrm{mM}$ hemin (MilliporeSigma), 30\% FBS, $1 \mathrm{U} / \mathrm{mL}$ recombinant human erythropoietin (rhEPO) (Amgen), $50 \mathrm{ng} / \mathrm{mL}$ recombinant mouse stem cell factor ( $\mathrm{rmSCF}$ ) (R\&D Systems; catalog 455-MC), and $5 \% \mathrm{vol} / \mathrm{vol}$ pokeweed mitogen mouse splenic cell conditioned medium. Colonies were scored after 6 days of incubation in $5 \% \mathrm{CO}_{2}$ and lowered $5 \% \mathrm{O}_{2}$ in a humidified chamber, and granulocyte-macrophage CFU (CFU-GM), erythrocyte burst-forming units (BFU-E), and granulocyte, erythrocyte, macrophage, and megakaryocyte colony-forming units (CFU-GEMM) were distinguished by morphology of colonies. The total numbers of colonies per femur were calculated. For high specific activity tritiated thymidine kill assays, BM cells were treated with $50 \mu \mathrm{Ci}$ high specific activity [3H]Tdr $(20 \mathrm{Ci} / \mathrm{mmol}$; DuPont NEN) at RT for 40 minutes and then washed twice prior to plating for HPC colony assays $(62,63)$. Note that this is currently the only means to assess the cycling status of functional populations of CFU-GM, BFU-E, and CFU-GEMM. 
Homing assays. Twelve million $\mathrm{BM}$ cells were harvested from young and old $\mathrm{C} 57 \mathrm{BL} / 6$ mice $\left(\mathrm{CD} 45.1^{-} \mathrm{CD} 45.2^{+}\right)$. Cells were injected i.v. into lethally irradiated (950 cGy, 24 hours prior) B6 Boy/J F1 $\left(\mathrm{CD} 45.1^{+} \mathrm{CD} 45.2^{+}\right)$recipient mice while under either hypoxic or ambient air conditions as previously described in the transplantation section. Eighteen hours following injection, BM from the recipient mice was collected and analyzed by flow cytometry for the presence of CD $45.1^{-}$ CD $45.2^{+}$LSK CD $150^{+}$cells (see Figure $3 \mathrm{G}$ for the experimental design).

Mass cytometry (CyTOF). For these studies, lineage depletion of mouse BM was performed with a Mouse Lineage Cell Depletion Kit (Miltenyi Biotec) following the manufacturer's protocol, using 2 sequential columns. The antibodies used in this study, along with their clones are listed in Supplemental Table 3. All preconjugated antibodies were purchased from DVS Sciences or Fluidigm. Several antibodies were conjugated in-house using the Maxpar Antibody Labeling Kit (Fluidigm). Each antibody was titrated to determine the optimal concentration for labeling of lineage-negative BM cells. After $\mathrm{BM}$ preparation, cells were treated with brefeldin A (MilliporeSigma) for 3 hours to inhibit protein transport. Following stimulation, cells were stained with Cell-ID Cisplatin viability stain (DVS Sciences, Fluidigm) for 2 minutes at RT. Cells were washed using stain wash $(0.1 \%$ BSA, 0.1\% Na-Azide, $10 \mathrm{nM}$ EDTA in $1 \times$ PBS) and then blocked using a Fc-receptor-blocking solution (BioLegend). Cells were stained with extracellular metal-labeled antibodies at $4^{\circ} \mathrm{C}$ for 30 minutes. After staining, cells were washed using stain wash and then fixed with $1.5 \%$ formaldehyde for 30 minutes. Ten-minute washes (×2) with Maxpar Perm-S Buffer (DVS Sciences, Fluidigm) were performed to permeabilize cells. Next, cells were stained with intracellular metal-labeled antibodies at $4^{\circ} \mathrm{C}$ for 30 minutes. Following incubation, cells were washed using stain wash and incubated overnight in 1:1000 Cell-ID Intercalator-Ir diluted in Maxpar Fix and Perm Buffer (DVS Sciences, Fluidigm). The next day, cells were washed once with stain wash and twice with Millipore water followed by resuspension in $1 \times$ EQ Calibration Beads (DVS Sciences, Fluidigm). Samples were analyzed on a CyTOF 2 mass cytometer (DVS, Sciences, Fluidigm). The bead signature was used to normalize raw CyTOF data before analysis with Cytobank software (Beckman Coulter).
viSNE analysis of normalized CyTOF events. Exported files were gated on singlet viable cells based on DNA labeling with iridium (Ir191/193), event length, and cisplatin (Pt195). Samples were gated on $\mathrm{Lin}^{-} \mathrm{Sca}^{+} \mathrm{CKit}^{+}$(LSK) CD48-CD150+ (LT-HSCs). These gated LSK $\mathrm{CD} 48^{-} \mathrm{CD} 150^{+}$cells were used to make viSNE plots and heatmaps with Cytobank software. Each antibody was further annotated on the viSNE plots to determine phenotypic differences between ambient air-acclimated and hypoxia-isolated LT-HSCs. The scale was the mean marker intensity of arcsinh-transformed values.

Statistics. Results are expressed as the mean values \pm SD or SEM as indicated in the figure legends. Unless otherwise indicated, 1-way ANOVA with a post hoc Tukey's multiple-comparison test was used for statistical analysis. Poisson statistical analysis was performed for the limiting dilution assays. A $P$ value less than 0.05 was considered statistically significant.

Study approval. All animal procedures were approved by the Committee on the Use and Care of Animals of the Indiana University School of Medicine.

\section{Author contributions}

MLC, SFM, and HEB conceived the research, designed and performed experiments, interpreted data, and wrote the manuscript. SC, BG, XH, AMG, CS, and JR performed experiments. EFS and CMO provided materials, helped design experiments, interpreted data, and helped revise the manuscript.

\section{Acknowledgments}

These studies were supported by NIH grants R35 HL 139599 (to HEB), R01 DK 109188 (to HEB and MLC), and U54 DK 106846 (to HEB and EFS).

Address correspondence to: Hal E. Broxmeyer or Maegan L. Capitano, Department of Microbiology and Immunology, Indiana University School of Medicine, 950 West Walnut Street, R2-302, Indianapolis, Indiana 46202, USA. Phone: 317.274.7510; Email: hbroxmey@iupui.edu (HEB). Phone: 317.274.7555; E-mail: mlcapit@iupui.edu (MLC).
1. Beerman I, Maloney WJ, Weissmann IL, Rossi DJ. Stem cells and the aging hematopoietic system. Curr Opin Immunol. 2010;22(4):500-506.

2. Geiger H, de Haan G, Florian MC. The ageing haematopoietic stem cell compartment. Nat Rev Immunol. 2013;13(5):376-389.

3. Kuranda K, et al. Age-related changes in human hematopoietic stem/progenitor cells. Aging Cell. 2011;10(3):542-546.

4. Miller JP, Allman D. Linking age-related defects in B lymphopoiesis to the aging of hematopoietic stem cells. Semin Immunol. 2005;17(5):321-329.

5. Rossi DJ, et al. Cell intrinsic alterations underlie hematopoietic stem cell aging. Proc Natl Acad Sci US A. 2005;102(26):9194-9199.

6. Florian MC, et al. Cdc42 activity regulates hematopoietic stem cell aging and rejuvenation. Cell Stem Cell. 2012;10(5):520-530.

7. Pang WW, et al. Human bone marrow hematopoietic stem cells are increased in frequency and myeloid-biased with age. Proc Natl Acad Sci U S A. 2011;108(50):20012-20017.
8. Rossi DJ, Jamieson CH, Weissman IL. Stems cells and the pathways to aging and cancer. Cell. 2008;132(4):681-696.

9. Signer RA, Montecino-Rodriguez E, Witte ON, McLaughlin J, Dorshkind K. Age-related defects in B lymphopoiesis underlie the myeloid dominance of adult leukemia. Blood. 2007;110(6):1831-1839.

10. Kaelin WG, Ratcliffe PJ, Semenza GL. Pathways for oxygen regulation and homeostasis: the 2016 Albert Lasker Basic Medical Research Award. JAMA. 2016;316(12):1252-1253.

11. Semenza GL. Dynamic regulation of stem cell specification and maintenance by hypoxia-inducible factors. Mol Aspects Med. 2016;47-48:15-23.

12. Broxmeyer HE, Cooper S, Rubin BY, Taylor MW. The synergistic influence of human interferon-gamma and interferon-alpha on suppression of hematopoietic progenitor cells is additive with the enhanced sensitivity of these cells to inhibition by interferons at low oxygen tension in vitro. Jimmunol. 1985;135(4):2502-2506.
13. Danet GH, Pan Y, Luongo JL, Bonnet DA, Simon MC. Expansion of human SCID-repopulating cells under hypoxic conditions. JClin Invest. 2003;112(1):126-135.

14. Lu L, Broxmeyer HE. Comparative influences of phytohemagglutinin-stimulated leukocyte conditioned medium, hemin, prostaglandin $\mathrm{E}$, and low oxygen tension on colony formation by erythroid progenitor cells in normal human bone marrow. Exp Hematol. 1985;13(10):989-993.

15. Rich IN, Kubanek B. The effect of reduced oxygen tension on colony formation of erythropoietic cells in vitro. Br J Haematol. 1982;52(4):579-588.

16. Smith S, Broxmeyer HE. The influence of oxygen tension on the long-term growth in vitro of haematopoietic progenitor cells from human cord blood. Br J Haematol. 1986;63(1):29-34.

17. Morrison SJ, Scadden DT. The bone marrow niche for haematopoietic stem cells. Nature. 2014;505(7483):327-334.

18. Nombela-Arrieta C, et al. Quantitative imaging of haematopoietic stem and progenitor cell localiza- 
tion and hypoxic status in the bone marrow microenvironment. Nat Cell Biol. 2013;15(5):533-543.

19. Bradley TR, Hodgson GS, Rosendaal M. The effect of oxygen tension on haemopoietic and fibroblast cell proliferation in vitro. JCell Physiol. 1978;97(3 pt 2 suppl 1):517-522.

20. Broxmeyer HE, Cooper S, Gabig T. The effects of oxidizing species derived from molecular oxygen on the proliferation in vitro of human granulocyte-macrophage progenitor cells. Ann N Y Acad Sci. 1989;554:177-184.

21. Broxmeyer HE, O'Leary HA, Huang X, Mantel C. The importance of hypoxia and extra physiologic oxygen shock/stress for collection and processing of stem and progenitor cells to understand true physiology/pathology of these cells ex vivo. Curr Opin Hematol. 2015;22(4):273-278.

22. Huang X, Trinh T, Aljoufi A, Broxmeyer HE. Hypoxia signaling pathway in stem cell regulation: good and evil. Curr Stem Cell Rep. 2018;4(2):149-157.

23. Mantel CR, et al. Enhancing hematopoietic stem cell transplantation efficacy by mitigating oxygen shock. Cell. 2015;161(7):1553-1565.

24. Ergen AV, Boles NC, Goodell MA. Rantes/ Ccl5 influences hematopoietic stem cell subtypes and causes myeloid skewing. Blood. 2012;119(11):2500-2509.

25. Capitano ML, Griesenauer B, Guo B, Cooper S, Paczesny S, Broxmeyer HE. The IL-33 Receptor/ ST2 acts as a positive regulator of functional mouse bone marrow hematopoietic stem and progenitor cells. Blood Cells Mol Dis. 2020;84:102435.

26. Chen $Y$, et al. Phorbol ester induced ex vivo expansion of rigorously-defined phenotypic but not functional human cord blood hematopoietic stem cells: a cautionary tale demonstrating that phenotype does not always recapitulate stem cell function. Leukemia. 2019;33(12):2962-2966.

27. Dorrell C, Gan OI, Pereira DS, Hawley RG, Dick JE. Expansion of human cord blood CD34(+) CD38(-) cells in ex vivo culture during retroviral transduction without a corresponding increase in SCID repopulating cell (SRC) frequency: dissociation of SRC phenotype function. Blood. 2000;95(1):102-110.

28. Beerman I, et al. Functionally distinct hematopoietic stem cells modulate hematopoietic lineage potential during aging by a mechanism of clonal expansion. Proc Natl Acad Sci U S A. 2010;107(12):5465-5470.

29. Kamminga LM, et al. Impaired hematopoietic stem cell functioning after serial transplantation and during normal aging. Stem Cells. 2005;23(1):82-92.

30. Huang X, Broxmeyer HE. Progress towards improving homing and engraftment of hematopoietic stem cells for clinical transplantation. Curr Opin Hematol. 2019;26(4):266-272.

31. Broxmeyer HE, et al. Transgenic expression of stromal cell-derived factor-1/CXC chemokine ligand 12 enhances myeloid progenitor cell sur- vival/antiapoptosis in vitro in response to growth factor withdrawal and enhances myelopoiesis in vivo. JImmunol. 2003;170(1):421-429.

32. Broxmeyer HE, et al. Stromal cell-derived factor-1/CXCL12 directly enhances survival/ antiapoptosis of myeloid progenitor cells through CXCR4 and G(alpha)i proteins and enhances engraftment of competitive, repopulating stem cells. JLeukoc Biol. 2003;73(5):630-638.

33. $\mathrm{Kim} \mathrm{CH}$, Broxmeyer HE. In vitro behavior of hematopoietic progenitor cells under the influence of chemoattractants: stromal cell-derived factor-1, steel factor, and the bone marrow environment. Blood.1998;91(1):100-110.

34. Ma Q, et al. Impaired B-lymphopoiesis, myelopoiesis, and derailed cerebellar neuron migration in CXCR4- and SDF-1-deficient mice. Proc Natl Acad Sci U S A. 1998;95(16):9448-9453.

35. Nagasawa T. The chemokine CXCL12 and regulation of HSC and B lymphocyte development in the bone marrow niche. Adv Exp Med Biol. 2007;602:69-75.

36. Lapidot T, Dar A, Kollet O. How do stem cells find their way home? Blood. 2005;106(6):1901-1910.

37. Nagasawa T. CXCL12/SDF-1 and CXCR4. Front Immunol. 2015;6:301.

38. Nagasawa T, et al. Defects of B-cell lymphopoiesis and bone-marrow myelopoiesis in mice lacking the CXC chemokine PBSF/SDF-1. Nature. 1996;382(6592):635-638.

39. Zou YR, Kottmann AH, Kuroda M, Taniuchi I, Littman DR. Function of the chemokine receptor CXCR4 in haematopoiesis and in cerebellar development. Nature. 1998;393(6685):595-599.

40. Broxmeyer HE. Enhancement of stem cell engraftment on a WHIM. JClin Invest. 2018;128(8):3240-3242.

41. Gao JL, et al. Cxcr4-haploinsufficient bone marrow transplantation corrects leukopenia in an unconditioned WHIM syndrome model. JClin Invest. 2018;128(8):3312-3318.

42. Liang Y, Van Zant G, Szilvassy SJ. Effects of aging on the homing and engraftment of murine hematopoietic stem and progenitor cells. Blood. 2005;106(4):1479-1487.

43. Krensky AM, Ahn YT. Mechanisms of disease: regulation of RANTES (CCL5) in renal disease. Nat Clin Pract Nephrol. 2007;3(3):164-170.

44. Sohy D, et al. Hetero-oligomerization of CCR2, CCR5, and CXCR4 and the protean effects of "selective" antagonists. J Biol Chem. 2009;284(45):31270-31279.

45. Calderwood SK, Murshid A, Prince T. The shock of aging: molecular chaperones and the heat shock response in longevity and aging - a mini-review. Gerontology. 2009;55(5):550-558.

46. Fan GC. Role of heat shock proteins in stem cell behavior. Prog Mol Biol Transl Sci. 2012;111:305-322.

47. McArdle A, et al. HSF expression in skeletal muscle during myogenesis: implications for failed regeneration in old mice. Exp Gerontol.
2006;41(5):497-500.

48. McArdle A, Dillmann WH, Mestril R, Faulkner JA, Jackson MJ. Overexpression of HSP70 in mouse skeletal muscle protects against muscle damage and age-related muscle dysfunction. FASEB J. 2004;18(2):355-357.

49. Delgado MD, León J. Myc roles in hematopoiesis and leukemia. Genes Cancer. 2010;1(6):605-616

50. Ghosh J, Kapur R. Role of mTORC1-S6K1 signaling pathway in regulation of hematopoietic stem cell and acute myeloid leukemia. Exp Hematol. 2017;50:13-21.

51. Yang A, Xiao X, Zhao M, LaRue AC, Schulte BA, Wang GY. Differential reponses of hematopoietic stem and progenitor cells to mTOR Inhibition. Stem Cells Int. 2015;2015:561404.

52. Cheng CW, et al. Prolonged fasting reduces IGF-1/PKA to promote hematopoietic-stemcell-based regeneration and reverse immunosuppression. Cell Stem Cell. 2014;14(6):810-823.

53 . Wang $X$, et al. TGF- $\beta 1$ negatively regulates the number and function of hematopoietic stem cells. Stem Cell Rep. 2018;11(1):274-287.

54. Jang Y-Y, Sharkis SJ. A low level of reactive oxygen species selects for primitive hematopoietic stem cells that may reside in the low-oxygen niche. Blood. 2007;110(8):3056-3063.

55. Chen F, Liu Y, Wong NK, Xiao J, So KF. Oxidative stress in stem cell aging. Cell Transplant. 2017;26(9):1483-1495.

56. Li X, et al. Mechanisms and rejuvenation strategies for aged hematopoietic stem cells. J Hematol Oncol. 2020;13(1):31.

57. Cai Q, Capitano M, Huang X, Guo B, Cooper S, Broxmeyer HE. Combinations of antioxidants and/or of epigenetic enzyme inhibitors allow for enhanced collection of mouse bone marrow hematopoietic stem cells in ambient air. Blood Cells Mol Dis. 2018;71:23-28.

58. Broxmeyer HE, Cooper S, Capitano ML. Enhanced collection of phenotyped and engrafting human cord blood hematopoietic stem cells at $4^{\circ} \mathrm{C}$. Stem Cells. 2020;38(10):1326-1331.

59. Wagner W, Horn P, Bork S, Ho AD. Aging of hematopo ietic stem cells is regulated by the stem cell niche. Exp Gerontol. 2008;43(11):974-980.

60. Kubo M, et al. Hypoxic preconditioning enhances angiogenic potential of bone marrow cells with aging-related functional impairment. Circ J. 2012;76(4):986-994.

61. Aljoufi A, Cooper S, Broxmeyer HE. Collection and processing of mobilized mouse peripheral blood at lowered oxygen tension yields enhanced numbers of hematopoietic stem cells. Stem Cell Rev Rep. 2020;16(5):946-953.

62. Capitano ML, et al. Secreted nuclear protein DEK regulates hematopoiesis through CXCR2 signaling. J Clin Invest. 2019;129(6):2555-2570.

63. Broxmeyer HE, et al. Dipeptidylpeptidase 4 negatively regulates colony-stimulating factor activity and stress hematopoiesis. Nat Med. 2012;18(12):1786-1796. 